\title{
Analysis of Degrees of Freedom under Mixture Gaussian Model in Cognitive Radio Systems
}

\author{
Ahsan-Abbas Ali ${ }^{\mathrm{a}}$, Shuangqing Wei ${ }^{\mathrm{a}, *}$ \\ a 3101 Patrick Taylor Hall, Louisiana State University, Baton Rouge, LA 70803, USA
}

\begin{abstract}
We propose a mixture-Gaussian model for a cognitive radio channel to analyze the interplay between the interference in the system and the degrees-of-freedom (DOF), i.e., the average number of channel uses per transmission frame, used by the secondary user (SU) for communications in the long run. In contrast to the conventional studies, we assume that the SU receiver (SU-RX) does not precisely know whether the primary-user (PU) transmitter is on or off. Due to this assumption the resulting interference channel is mixture-Gaussian. Our objective is to find the optimal sensing threshold and sensing time for the signal detector used by the SU transmitter (SU-TX). Our formulation of the optimization problem reflects the trade-off between SU-TX's DOF for communications and that for detection. Both the DOFs affect PU's interference to SU, and SU's interference to PU. The latter interference causes PU performance degradation, which is kept within tolerable range as a constraint. As a further contribution, we define interference regimes for SU performance on the basis of PU transmission power level. We also address the scenario when PU receiver uses the nearest neighbor decoding while wrongly anticipating that the channel is Gaussian. Finally, we demonstrate that even if SU-TX's signal detector performs suboptimally, SU can still achieve the optimal detector's performance in the high interference regime by adjusting the sensing parameters. Keywords: cognitive radio, degrees of freedom, interference, mutual information, energy detection, envelope detection.
\end{abstract}

\section{Introduction}

The users of sophisticated broadband wireless services are increasing day by day, thereby raising the demand of these services. Therefore, already established networks are expanding their resources and the new service providers are establishing their infrastructure. This gives rise to the problem

5 of scarce bandwidth resources. Since most of the available spectrum has already been licensed, there is almost no room left for accommodating the new demands. There are studies like [1] which show that vast regions of licensed spectrum are underutilized. These are called white-spaces. A

\footnotetext{
${ }^{*}$ Corresponding author, phone: +1 (225) 578-5536

Email addresses: ahsabali@gmail.com (Ahsan-Abbas Ali), swei@ece.lsu.edu (Shuangqing Wei )
} 
prospective solution to the problem of scarce bandwidth resources is to use these white spaces for the new wireless systems instead of issuing the new licenses. The device that can help us achieve this goal of utilizing the unused channel is the cognitive radio 2 , 3. It is a radio that can sense and learn, as the word 'cognitive' indicates. It has intelligent capabilities to sense the communication activities over the channel and looks for the opportunities available for itself.

In a cognitive radio $(\mathrm{CR})$ based system, there are two categories of users. One of them is called the primary user (PU) and the other is called the secondary user (SU). PU's are the users which possess the license for using the channel and can use it for communications at anytime. On the other hand, SU's are the unlicensed users and can only use the channel for communications when it is idle. These SU's form the nodes of the cognitive radio network and are intelligent enough to sense whether the channel is being used by a PU or not. This is referred to as the channel sensing ability of the SU. Also, SU should leave an occupied channel whenever a PU starts transmission using that channel.

We consider a simple cognitive radio system consisting of two transmitter-receiver pairs, one for the primary user PU and the other for the secondary user SU. They have a common frequency channel to use for communications and SU communicates whenever the channel is sensed idle. In order to find out whether the channel is occupied by a PU signal or not, SU transmitter uses a signal 25 detector, e.g., the energy detector. For this system, the main design problem is to find the optimal sensing time and threshold for the detector used by SU transmitter. Here we would like to mention an important remark, i.e., for the rest of the discussion, the signal detector of $S U$ refers to the signal detector used by the SU transmitter. The optimality criterion is to maximize the SU performance while keeping the detection probability above an appropriate threshold level. This criterion is also solled the sensing-throughput trade-off for SU.

Note that in this study, coherence means the availability of the channel state information (CSI) to the receiver [4, 5]. In our model, CSI for the SU receiver is the precise knowledge of the PU transmitter's transmission state, i.e., whether it is on or off. The sensing-throughput trade-off and analysis models have been explored in [6, 7, 8, 9, 10, 11, 12, 13, 14, 15, 16, 17, 18, 19], under an assumption that SU receiver operates coherently with both SU and PU transmitters, i.e., SU receiver precisely knows if $\mathrm{SU}$ and $\mathrm{PU}$ transmitters are on or off. We call this the conventional model. However, in this paper, we consider a more practical scenario where SU receiver operates coherently with SU transmitter but incoherently with PU transmitter, as in our previous work [20]. This means that SU receiver precisely knows if SU transmitter is on or off, but it does not know the 40 same about PU transmitter. This model appropriately incorporates the interference experienced by both PU and SU systems and is practically more rigorous than the conventional model. To the best of our knowledge, despite such significance, this model has never been studied.

The assumption that whether the SU receiver operates coherently or incoherently with the PU 
transmitter, determines the nature of the interference channel in the system model. In the conventional model, due to the coherent operation of SU receiver and PU transmitter, the underlying channel is a regular Gaussian interference channel. On the other hand, in our model, due to the incoherent operation of SU receiver and PU transmitter, the resulting interference channel is mixtureGaussian, as explained in Section 2, Due to the mixture-Gaussian nature of the interference channel, the optimal sensing time and threshold design problem can be better elaborated with the help of a novel concept of a trade-off, that we propose formally in Section 5.1 as the trade-off between the degrees of freedom available to SU for communications and the interference experienced by SU due to PU, rather than that of the conventional sensing-throughput trade-off. In this study, the degrees of freedom available to SU for communications quantify the average number of channel-uses per transmission frame, which is used by SU for communications in the long run, i.e., for infinitely large number of transmission frames, as explained in detail in Section 5.1. We properly define and elaborate the novel concept of the trade-off in Section 5.1, and before proceeding to it, we also build the required background in the subsequent sections, in order to help the reader understand the concept nicely.

Due to the incoherence assumption between SU receiver and PU transmitter, the analysis becomes more challenging. The first challenge is that the interference channel becomes mixture Gaussian that requires the evaluation of the entropy of mixture Gaussian random variables, which does not have a closed form solution. The second challenge is the non-linear interdependence of quite a few parameters. Thus, the optimization problem under consideration becomes quite complicated and cannot be solved using the conventional optimization techniques, as explained in Section 3.2 . Also, it does not have a closed form solution, and therefore, we develop numerical algorithms to solve it.

Another loose end in the conventional studies is that an arbitrarily high detection-probability threshold is selected for the detection-probability constraint. In order to tie up this loose end, we select a meaningful value for this threshold which ensures that the PU performance degradation remains within a tolerable range. This requires us to explicitly compute PU performance. While evaluating PU performance, we assume that PU receiver does not know the state of SU transmitter, i.e., PU receiver also operates incoherently with SU transmitter, in the same way as we assume that SU receiver operates incoherently with PU transmitter. Note that a degradation in PU performance is caused by the interference due to SU, when the detection probability is less than 1 .

75 In 20] the sensing-throughput trade-off was explored for a binary coded communication system, the optimization problem was formulated only and some special cases about sensing and throughput trade-off were investigated. In this paper, we extend our previous work in [20] by solving the optimization problem for a more general coded case. As a further novel contribution, we identify a performance inefficiency region for SU at a very high detection probability, where SU performance 
drastically decreases with increase in detection probability. We call this the energy detector's inefficiency region and is abbreviated as EDI region. We also identify the interference regimes for SU performance on the basis of PU transmission power level $\mathcal{P}_{\mathrm{PU}}$. Our study reveals that the tolerance level of PU performance can be exploited to increase the SU performance by sacrificing the detection probability, but only for the low interference regime for $S U$. In this regime, the PU transmission power is weak and at a level lower than some threshold, such that the SU performance increases with decrease in detection probability. On the other hand, for the high interference regime in which the PU power is stronger, the SU cannot sacrifice the detection probability at all. This is because the interference caused by PU is so high that the SU performance decreases with decrease in detection probability for the high interference regime. Therefore, during this regime, the detection probability needs to be kept at the maximum level for the non-EDI region, such that it is greater than the required minimum threshold but small enough to avoid the EDI region. This change in the interference regimes for the SU performance is not revealed by the conventional model, and can only be observed under the mixture Gaussian model proposed in our paper. Note that we also provide the values of the thresholds for the PU transmission power that determine the regime change for the SU performance, based on the numerical results. Furthermore, the discovery of such interesting trends is neither trivial nor straightforward. Our adopted optimization framework, explained in Sections 3.2 and 3.3 helps us identify these trends in SU performance. We finally demonstrate that even if the signal detector of SU performs suboptimally, SU can still achieve the optimal detector's performance level in the high interference regime for SU just by adjusting the sensing parameters accordingly. Our results provide a guideline for the design of a practical cognitive radio system, based on the transmission-power levels of the users, to achieve the maximum secondary-user's performance by keeping the primary-user's performance-degradation within a given tolerable range.

Since we are interested in revealing the effect of the mixture-Gaussian interference on the degreesof-freedom and interference trade-off, we thus keep our model simple and do not incorporate fading and exact information of receivers' locations. However, these features are required for a more precise modeling of interference that is left for future exploration. Even in the absence of these features, the computational complexity of our problem is very high, due to the incoherence assumption between SU receiver and PU transmitter, which results in a mixture Gaussian interference channel and nonlinear interdependence of quite a few parameters in our system model, as explained in Section 3.2 . Also recall that we only consider a single point-to-point communication link for both PU and SU in our system model. Even this simple model poses analytical challenges and reveals interesting results, as elaborated in this study. However, this basic model can be used as a building block for a more sophisticated model of cognitive radio networks, which may consist of large number of nodes and communication links among them. Such complicated model considerations are left for future work. 
Part of this study has been presented in 21. In this paper we further elaborate interesting aspects of the problem, which are not addressed in [21]. In particular, we discuss the conventional model in detail and elaborate the fundamental difference between the information theoretic framework of our model and that of the conventional one in Section 4. In Section 6 we discuss how the analysis tem model, SU-TX and SU-RX represent the secondary user transmitter and receiver, respectively, whereas, PU-TX and PU-RX represent the primary user transmitter and receiver respectively. In

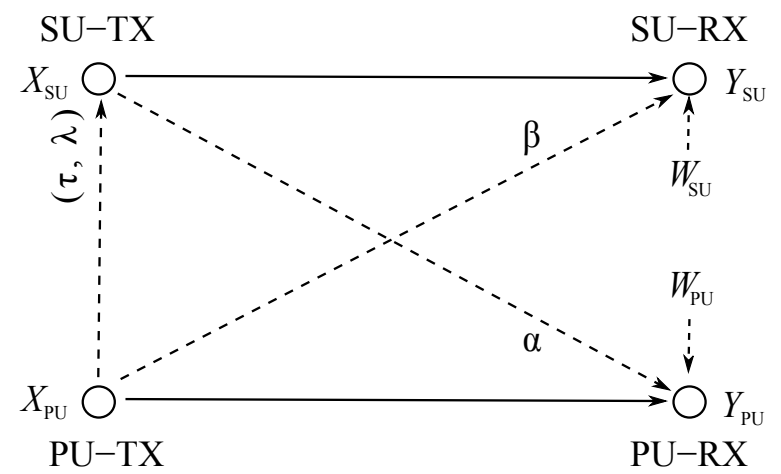

Figure 1: System Model

this paper we consider periodic sensing that enables SU-TX to remain aware of the channel status. This is achieved by using a frame structure $6,18,19,20$. In this structure, each frame consists of a sensing period and a transmission period, while the overall length of the frame is fixed. At the 


\begin{tabular}{|c|c|}
\hline Symbol & Definition \\
\hline$N$ & Total number of channel uses in one frame \\
\hline$N_{\mathrm{s}}$ & Number of channel uses in the sensing period of SU frame \\
\hline$N_{\mathrm{c}}$ & Number of channel uses in the communication period of SU frame \\
\hline$\tau$ & Portion of the total frame-time that is used by SU-TX for sensing \\
\hline$\lambda$ & SU-TX sensing threshold \\
\hline$\tilde{\tau}_{0}$ & Optimal solution for $\tau$ \\
\hline$\tilde{\lambda}_{0}$ & Optimal solution for $\lambda$ \\
\hline$\pi_{0}$ & $\operatorname{Pr}$ (PU-TX is off for the time of entire frame) \\
\hline$\pi_{1}$ & $\operatorname{Pr}(\mathrm{PU}-\mathrm{TX}$ is on for the time of entire frame) \\
\hline$\psi_{1}$ & $\operatorname{Pr}$ (SU-TX is on for the communication period of SU frame) \\
\hline$X_{\mathrm{PU}}$ & Transmitted symbol during a single channel use by PU-TX \\
\hline$X_{\mathrm{SU}}$ & Transmitted symbol during a single channel use by SU-TX \\
\hline$W_{\mathrm{PU}}$ & Noise component in the received symbol for PU-RX during a single channel use \\
\hline$W_{\mathrm{SU}}$ & Noise component in the received symbol for SU-RX during a single channel use \\
\hline$Y_{\mathrm{PU}}$ & Received symbol for PU-RX during a single channel use \\
\hline$Y_{\mathrm{SU}}$ & Received symbol for SU-RX during a single channel use \\
\hline$\alpha$ & Coupling coefficient for PU-RX \\
\hline$\beta$ & Coupling coefficient for SU-RX \\
\hline$P_{\alpha}$ & $\operatorname{Pr}(\alpha=1)$ \\
\hline$P_{\beta}$ & $\operatorname{Pr}(\beta=1)$ \\
\hline$Z_{\mathrm{PU}}$ & $=W_{\mathrm{PU}}+\alpha X_{\mathrm{SU}}$ \\
\hline$Z_{\mathrm{SU}}$ & $=W_{\mathrm{SU}}+\beta X_{\mathrm{PU}}$ \\
\hline $\mathcal{P}_{\mathrm{PU}}$ & PU transmission power level \\
\hline$\hat{\mathcal{P}}_{\mathrm{PU}}$ & $\mathcal{P}_{\text {PU }}$ threshold \\
\hline $\mathcal{P}_{\mathrm{SU}}$ & SU transmission power level \\
\hline$P_{\mathrm{F}}$ & False alarm probability \\
\hline$\tilde{P}_{\mathrm{F} 0}$ & Value of $P_{\mathrm{F}}$ achieved at optimal solution \\
\hline$P_{\mathrm{D}}$ & Detection probability \\
\hline$\tilde{P}_{\mathrm{D}}$ & A given fixed value of $P_{\mathrm{D}}$ \\
\hline$P_{\mathrm{D}_{\mathrm{T}}}$ & The constraint threshold for $P_{\mathrm{D}}$ \\
\hline$P_{\mathrm{D}_{\mathrm{T} 2}}$ & Value of $P_{\mathrm{D}}$ at which EDI region starts \\
\hline$\tilde{P}_{\mathrm{D} 0}$ & Value of $P_{\mathrm{D}}$ achieved at optimal solution \\
\hline$H$ & Actual channel-state variable \\
\hline$\hat{H}$ & Decision channel-state variable \\
\hline$I_{\mathrm{SU}-\mathrm{N}}$ & Mutual information for SU system for the entire frame \\
\hline$\hat{I}_{\mathrm{SU}-\mathrm{N}}$ & SU information rate for a single channel use \\
\hline$\tilde{I}_{\mathrm{SU}-\mathrm{N}}$ & $=\max _{\tau, \lambda} I_{\mathrm{SU}-\mathrm{N}}$, s.t. $P_{\mathrm{D}}=\tilde{P}_{\mathrm{D}}$ \\
\hline$\tilde{\hat{I}}_{\mathrm{SU}-\mathrm{N}}$ & Value of $\hat{I}_{\mathrm{SU}-\mathrm{N}}$, when the $\mathrm{SU}$ information rate is $\tilde{I}_{\mathrm{SU}-\mathrm{N}}$ \\
\hline$\nu_{1}$ & Upper bound on $I_{\mathrm{SU}-\mathrm{N}}$ for perfect detection \\
\hline$I_{\mathrm{PU}-\mathrm{N}}$ & Mutual information for PU system for the entire frame \\
\hline$\tilde{I}_{\mathrm{PU}-\mathrm{N}}$ & $=I_{\mathrm{PU}-\mathrm{N}}$, s.t. $I_{\mathrm{SU}-\mathrm{N}}=\tilde{I}_{\mathrm{SU}-\mathrm{N}}$ \\
\hline$\hat{I}_{\mathrm{PU}-\mathrm{Ns}}$ & PU information rate for a single channel use during the sensing period of SU \\
\hline$\hat{I}_{\mathrm{PU}-\mathrm{Nc}}$ & PU information rate for a single channel use during the communication period of SU \\
\hline$\Delta_{\mathrm{PU}}$ & PU performance degradation factor \\
\hline$\Delta_{\mathrm{T}}$ & Maximum allowed value of $\Delta_{\mathrm{PU}}$ \\
\hline$\tilde{\Delta}_{0}$ & Value of $\Delta_{\mathrm{PU}}$ achieved at optimal solution \\
\hline
\end{tabular}

Table 1: Table of Symbols 
end of each sensing period, the secondary transmission starts when the PU channel is considered as idle by SU-TX. Otherwise, SU-TX will wait until the next frame to sense the licensed channel again before any secondary usage. Thus we assume encoding of SU system is based on this frame structure such that each frame consists of, say, $N$ channel uses and the information rate is achieved by encoding for infinitely large number of such frames.

We define a parameter $\tau$ as the portion of the total frame-time that is used by SU-TX for sensing the occupation of the channel by the PU. For SU-TX, $N_{\mathrm{s}}=\tau N$ represents the number of channeluses in a frame out of a total of $N$ that are used for sensing and is therefore called the sensing period. While $N_{\mathrm{c}}=N-N_{\mathrm{s}}=(1-\tau) N$ represents the number of channel-uses in a frame used for communications whenever there is a transmission opportunity for SU-TX and is thus called the communication period [6, 18, 19, 20].

The encoding of PU system is also based on a frame structure with $N$ channel uses and the information rate is thus achievable for infinitely large number of transmissions of such frames. We assume that PU-TX communicates data to PU-RX in an on-and-off manner in the form of frames over the given frequency channel. This on-and-off operation of PU-TX is modeled such that for every frame of $N$ channel uses PU-TX is either off for the time of entire frame with probability $\pi_{0}$ or it is on for the time of entire frame with probability $\pi_{1}=1-\pi_{0}$. It is assumed that there is a precedent learning or estimation phase where long-time scale measurements are taken to estimate $\pi_{1}$. Such learning or estimation problem is beyond the scope of this paper, which will be further investigated and presented in our future works.

Let $X_{\mathrm{PU}}$ and $X_{\mathrm{SU}}$ denote the independent transmitted symbols during a single channel use by PU-TX and SU-TX respectively while they access the channel. Similarly, $Y_{\mathrm{PU}}$ and $Y_{\mathrm{SU}}$ denote the corresponding received symbols for PU-RX and SU-RX respectively. All communication channels in the system are assumed to be additive white Gaussian noise channels. Let $W_{\mathrm{PU}}$ and $W_{\mathrm{SU}}$ denote the independent noise components in the received symbols for PU-RX and SU-RX respectively. Both these noise components are Gaussian random variables having zero mean and unit variance. Also the transmitted signal and the noise, and therefore the received signal, over any given channel-use are independent but distributed identically to those over all the other channel-uses.

We assume that PU-TX and PU-RX have no knowledge about the existence of SU-TX and SU-RX in the system. Therefore, perceiving a simple additive Gaussian noise channel, PU-TX transmits a Gaussian distributed symbol $X_{\mathrm{PU}}$ over each single channel use thereby attempting to gain a maximum capacity. Note, in this work we are not aiming at finding the optimal probability distributions for $X_{\mathrm{PU}}$ and $X_{\mathrm{SU}}$ that can achieve the channel capacity in the presence of interference. Therefore, we assume both as Gaussian random variables. Thus, the transmitters are assumed to possess a Gaussian code-book. The transmitted symbols are real Gaussian random variables each of which has a zero mean and a variance that is equal to the average power constraint of the transmitter. 
We use $\mathcal{P}_{\mathrm{PU}}$ and $\mathcal{P}_{\mathrm{SU}}$ to denote the average power constraints for PU-TX and SU-TX respectively.

We adopt a channel sensing model for SU-TX that is based on the energy detection scheme. The false alarm probability $P_{\mathrm{F}}$ and the detection probability $P_{\mathrm{D}}$ for our energy detector based sensing model are given by, 22], 23], 20], $P_{F}=\frac{\Gamma\left(N_{S} / 2, \lambda / 2\right)}{\Gamma\left(N_{S} / 2\right)}$ and $P_{D}=\frac{\Gamma\left(N_{S} / 2, \frac{\lambda}{2\left(1+\mathcal{P}_{\mathrm{PU}}\right)}\right)}{\Gamma\left(N_{S} / 2\right)}$. Here $\lambda$ is the sensing threshold and, $\Gamma($.$) and \Gamma(.,$.$) are respectively the complete and upper-incomplete Gamma$ functions [24].

The received signals at PU-RX and SU-RX are given by $Y_{\mathrm{PU}}=X_{\mathrm{PU}}+W_{\mathrm{PU}}+\alpha X_{\mathrm{SU}}$ and $Y_{\mathrm{SU}}=X_{\mathrm{SU}}+W_{\mathrm{SU}}+\beta X_{\mathrm{PU}}$ respectively. Here, $X_{\mathrm{PU}}$ and $X_{\mathrm{SU}}$ are Gaussian distributed with probability density functions $(\mathrm{PDFs}) \mathcal{N}\left(0, \mathcal{P}_{\mathrm{PU}}\right)$ and $\mathcal{N}\left(0, \mathcal{P}_{\mathrm{SU}}\right)$ respectively, while $W_{\mathrm{PU}}$ and $W_{\mathrm{SU}}$ both are Gaussian distributed with $\operatorname{PDF} \mathcal{N}(0,1)$. The random variables, $\alpha$ and $\beta$, are called the coupling coefficients. They appear because of the mutual interference between PU and SU users. We consider this interference in our system model because we assume that the SU-RX does not know the state of the channel access by PU-TX. Parameters $\alpha$ and $\beta$ are Bernoulli random variables having a value 0 or 1 . These coefficients can be described by the probabilities, $P_{\alpha}=\operatorname{Pr}(\alpha=1)$ and $P_{\beta}=\operatorname{Pr}(\beta=1)$, respectively, which are given by $P_{\alpha}=\operatorname{Pr}(\hat{H}=0 \mid H=1)=1-P_{\mathrm{D}}$ and $P_{\beta}=\operatorname{Pr}(H=1 \mid \hat{H}=0)=\frac{\pi_{1}\left(1-P_{D}\right)}{\pi_{0}\left(1-P_{F}\right)+\pi_{1}\left(1-P_{D}\right)}$. Here $\hat{H}$ and $H$ denote the decision and actual channel state variables, respectively, with 0 representing an idle primary channel and 1 for a busy primary channel.

Using Bayes rule, the PDF of $Y_{\mathrm{PU}}$ is

$$
\begin{aligned}
f_{\mathrm{Y}_{\mathrm{PU}}}(x)=\left(1-P_{\alpha}\right) & \times \mathcal{N}\left(x ; 0,1+\mathcal{P}_{\mathrm{PU}}\right) \\
& +P_{\alpha} \times \mathcal{N}\left(x ; 0,1+\mathcal{P}_{\mathrm{PU}}+\mathcal{P}_{\mathrm{SU}}\right),
\end{aligned}
$$

and that of $Y_{\mathrm{SU}}$ is

$$
\begin{aligned}
f_{\mathrm{Y}_{\mathrm{SU}}}(x)=\left(1-P_{\beta}\right) & \times \mathcal{N}\left(x ; 0,1+\mathcal{P}_{\mathrm{SU}}\right) \\
& +P_{\beta} \times \mathcal{N}\left(x ; 0,1+\mathcal{P}_{\mathrm{PU}}+\mathcal{P}_{\mathrm{SU}}\right) .
\end{aligned}
$$

It is evident from (1) and (2) that, because of the mutual interference between PU and SU the unwanted disturbance component in the received symbols have mixture Gaussian PDFs instead of pure Gaussian. Therefore we cannot use the well known result for the Gaussian channel capacity, $\log (1+\mathrm{SNR})$, as used for the conventional model where the unwanted component is simply Gaussian [6]. We therefore need to re-evaluate the information rates that are achievable over the resulting mixture Gaussian channel, in order to analyze the system performance.

For both SU-RX and PU-RX, the decoding schemes are assumed to be optimal, i.e., the Maximum Likelihood (ML) decoding. This assumption of using optimal decoding will ensure that the mutual 
information rates in our model are achievable and therefore considered as valid metrics for system throughput. But, it should be noted that ML decoding requires knowledge of channel statistics at the receiver and is complicated to implement for a non-Gaussian channel, as is our case. Later in Section 6, we comment on the effect of adopting a robust decoding scheme for PU-RX namely the nearest neighbor decoding [25].

\section{Problem Formulation and Solution Procedure}

\subsection{Performance Metrics:}

The performance metric for the SU system is the mutual information which quantifies the achievable communication rate per transmission frame, between SU-TX and SU-RX, when SU-RX uses the optimum maximum likelihood decoder, in the presence of an uncertainty of the PU channel state averaged over the long run, i.e., over infinitely large number of transmissions. Note that the PU channel state in our study represents whether the PU-TX is on or off. We assume that SU-RX does not have the knowledge of this PU channel state which causes interference in the SU system. Such a situation with a lack of knowledge on the channel quality status is called incoherent receiver operation [4, 5]. Similarly, the performance metric for the PU system is the mutual information which quantifies the achievable communication rate per transmission frame, between PU-TX and PU-RX, in the presence of an uncertainty of the SU channel state averaged over the long run. Recall that the encoding of the $\mathrm{SU}$ and PU systems is based on a frame structure with $N$ channel uses. The channel over each frame varies between good and bad, where good means no interference from another transmitter, and bad the opposite. The achievable rate is attainable through the encoding over a large number of frames to average out such channel uncertainties without delay constraints. If there is a memory between good and bad conditions, we essentially deal with the Giblert-Elliott channels [5], and such memory is essentially caused by the memory between on and off of PU-TX. In this study, we assume that PU-TX is either off over the entire frame with probability $\pi_{0}$, or it is on with probability $\pi_{1}=1-\pi_{0}$, without memory. Therefore, the mutual information for the PU system over $N$ channel uses of the entire frame is given by

$$
I_{\mathrm{PU}-\mathrm{N}}=\pi_{1}\left[N_{\mathrm{s}} \hat{I}_{\mathrm{PU}-\mathrm{Ns}}+N_{\mathrm{c}} \hat{I}_{\mathrm{PU}-\mathrm{Nc}}\right]
$$

where $\hat{I}_{\mathrm{PU}-\mathrm{Ns}}$ is the mutual information for PU over one channel use during the sensing period of SU-TX and $\hat{I}_{\mathrm{PU}-\mathrm{Nc}}$ is the mutual information for PU over one channel use during the communication period of SU-TX. During the sensing period, SU-TX is always silent and the PU channel is simply an Additive Gaussian Noise (AGN) channel therefore $\hat{I}_{\mathrm{PU}-\mathrm{Ns}}=(1 / 2) \log \left(1+\mathcal{P}_{\mathrm{PU}}\right)$. On the other hand, during the communication period SU-TX may transmit if it detects an opportunity and thus 
the PU channel is an Additive Mixture Gaussian (AMG) channel. Therefore,

$$
\hat{I}_{\mathrm{PU}-\mathrm{Nc}}=h\left(Y_{\mathrm{PU}}\right)-h\left(Y_{\mathrm{PU}} \mid X_{\mathrm{PU}}\right)=h\left(Y_{\mathrm{PU}}\right)-h\left(Z_{\mathrm{PU}}\right),
$$

where $h(X)=-\int f_{\mathrm{X}}(x) \log \left[f_{\mathrm{X}}(x)\right] d x$ is the differential entropy of random variable $X$ having PDF $f_{\mathrm{X}}(x)$ and $Z_{\mathrm{PU}}=W_{\mathrm{PU}}+\alpha X_{\mathrm{SU}}$ is a mixture Gaussian random variable with PDF

$$
\begin{aligned}
f_{\mathrm{Z}_{\mathrm{PU}}}(x)=\left(1-P_{\alpha}\right) & \times \mathcal{N}(x ; 0,1) \\
& +P_{\alpha} \times \mathcal{N}\left(x ; 0,1+\mathcal{P}_{\mathrm{SU}}\right) .
\end{aligned}
$$

SU system only communicates during the communication period whenever SU-TX detects an opportunity. Therefore, the mutual information for the SU system over $N$ channel uses of the entire frame is given by

$$
I_{\mathrm{SU}-\mathrm{N}}=\psi_{1} N_{\mathrm{c}} \hat{I}_{\mathrm{SU}-\mathrm{Nc}}
$$

Here the product term $\psi_{1} N_{\mathrm{c}}$ represents the degrees of freedom available to $\mathrm{SU}$ for communications, which quantify the average number of channel-uses per transmission frame used by SU for communications, in the long run. Note that $\psi_{1}=\operatorname{Pr}(\mathrm{SU}$ is on $)=\left(1-P_{\mathrm{F}}\right) \pi_{0}+\left(1-P_{\mathrm{D}}\right) \pi_{1}$ and $\hat{I}_{\mathrm{SU}-\mathrm{Nc}}$ is the mutual information for SU over one channel use during the communication period, given by

$$
\hat{I}_{\mathrm{SU}-\mathrm{Nc}}=h\left(Y_{\mathrm{SU}}\right)-h\left(Y_{\mathrm{SU}} \mid X_{\mathrm{SU}}\right)=h\left(Y_{\mathrm{SU}}\right)-h\left(Z_{\mathrm{SU}}\right),
$$

where $Z_{\mathrm{SU}}=W_{\mathrm{SU}}+\beta X_{\mathrm{PU}}$ is a mixture Gaussian random variable with $\mathrm{PDF}$

$$
\begin{aligned}
f_{\mathrm{Z}_{\mathrm{SU}}}(x)=\left(1-P_{\beta}\right) & \times \mathcal{N}(x ; 0,1) \\
& +P_{\beta} \times \mathcal{N}\left(x ; 0,1+\mathcal{P}_{\mathrm{PU}}\right) .
\end{aligned}
$$

\subsection{Optimization Problem and Procedure for Solution:}

A low detection probability requires small sensing time and thus corresponds to high degrees of freedom available to SU for communications. At the same time, it causes high interference for both $\mathrm{SU}$ and PU that results in a degradation in performance of both systems, and vice-versa. Therefore, variations in the sensing time and threshold affect the detection probability which incurs both loss and gain at the same time for SU, thereby indicating the need of a trade-off for SU performance. Our objective is to find the optimal sensing threshold $\lambda$ and sensing time $\tau$ that will maximize the $\mathrm{SU}$ performance and at the same time keep the detection quality within a suitable range. Thus, our objective is to find the optimal $\tau$ and $\lambda$ that will maximize the mutual information $I_{\mathrm{SU}-\mathrm{N}}$ under the 
constraint that $P_{\mathrm{D}}$ will not drop below a given threshold $P_{\mathrm{D}_{\mathrm{T}}}$, i.e.,

$$
\max _{\tau, \lambda} I_{\mathrm{SU}-\mathrm{N}}, \text { s.t. } P_{\mathrm{D}} \geq P_{\mathrm{D}_{\mathrm{T}}}
$$

Based on our discussion in Section 2 and 3.1. we can express the objective function in (9) in a more elaborative form as follows

$$
\max _{\tau, \lambda} \psi_{1}\left(P_{\mathrm{D}}(\tau, \lambda), P_{\mathrm{F}}(\tau, \lambda)\right) \cdot(1-\tau) N \cdot \int_{x \in \mathbb{R}} g\left(x, P_{\mathrm{D}}(\tau, \lambda), P_{\mathrm{F}}(\tau, \lambda)\right) d x, \text { s.t. } P_{\mathrm{D}}(\tau, \lambda) \geq P_{\mathrm{D}_{\mathrm{T}}}
$$

Note that $g\left(x, P_{\mathrm{D}}, P_{\mathrm{F}}\right)=f_{\mathrm{Y}_{\mathrm{SU}}}(x)-f_{\mathrm{Z}_{\mathrm{SU}}}(x)$, whereas the integral $\int g\left(x, P_{\mathrm{D}}, P_{\mathrm{F}}\right) d x=\hat{I}_{\mathrm{SU}-\mathrm{Nc}}$ is a difference of entropies of mixture Gaussian random variables, as shown in (7), which does not have a closed form solution and needs to be evaluated numerically [26. Moreover, as explained in Section 2 . $\psi_{1}$ in 10 and the distributions of the mixture Gaussian variables in our model are functions of false alarm and detection probabilities given by $P_{F}=\frac{\Gamma\left(N_{S} / 2, \lambda / 2\right)}{\Gamma\left(N_{S} / 2\right)}$ and $P_{D}=\frac{\Gamma\left(N_{S} / 2, \frac{\lambda}{2\left(1+\mathcal{P}_{\mathrm{PU})}\right.}\right)}{\Gamma\left(N_{S} / 2\right)}$. Recall from Section 2 that these probabilities are ratios of Gamma functions which are integrals without closed form solution [24]. Due to this analytical complexity of problem [9], we cannot use the standard analytical methods for optimization. Therefore, in this paper, we develop a numerical framework to solve the optimization problem (9), which is discussed in Section 5 . The framework includes the numerical evaluation of entropy of mixture Gaussian distributions and the numerical solution of problem (9). Here, in this Section, we present our two step optimization procedure that is used in the numerical framework. This optimization procedure enables us to quantify the SU performance as a function of a single variable, instead of two variables $\tau$ and $\lambda$, as explained in the subsequent paragraph. This helps generate SU performance results that are easy to visualize and analyze, and also present useful insights, as discussed in Section 5 .

In the first step of optimization, we fix $P_{\mathrm{D}}$ to some value say $\tilde{P}_{\mathrm{D}}$. For a given $P_{\mathrm{D}}, I_{\mathrm{SU}-\mathrm{N}}$ varies with $P_{\mathrm{F}}$, as detailed in Sections 2 and 3 . So, we maximize $I_{\mathrm{SU}-\mathrm{N}}$ over all such $\tau$ and $\lambda$ for which $P_{\mathrm{D}}=\tilde{P}_{\mathrm{D}}$. This first step results in the maximum values of $I_{\mathrm{SU}-\mathrm{N}}$ for each $\tilde{P}_{\mathrm{D}}$, which we represent as $\tilde{I}_{\mathrm{SU}-\mathrm{N}}$, given by

$$
\tilde{I}_{\mathrm{SU}-\mathrm{N}}=\max _{\tau, \lambda} I_{\mathrm{SU}-\mathrm{N}}, \text { s.t. } P_{\mathrm{D}}=\tilde{P}_{\mathrm{D}}
$$

$\tilde{I}_{\mathrm{SU}-\mathrm{N}}$ is thus a function of $\tilde{P}_{\mathrm{D}}$. Note, that $\tilde{P}_{\mathrm{D}}$ is used only for expressing the mathematical formulation (11), and that $\tilde{P}_{\mathrm{D}}$ and $P_{\mathrm{D}}$ are interchangeable everywhere in our analysis. In the second step of our optimization procedure, we select the maximum $\tilde{I}_{\mathrm{SU}-\mathrm{N}}$ such that $\tilde{P}_{\mathrm{D}} \geq P_{\mathrm{D}_{\mathrm{T}}}$ as shown in 12 .

$$
\max _{\tilde{P}_{\mathrm{D}}} \tilde{I}_{\mathrm{SU}-\mathrm{N}}, \text { s.t. } \tilde{P}_{\mathrm{D}} \geq P_{\mathrm{D}_{\mathrm{T}}}
$$


This concludes the optimization procedure. Note that the two step optimization procedure enables us to quantify the SU performance in terms of $\tilde{I}_{\mathrm{SU}-\mathrm{N}}$ which is a function of a single variable $\tilde{P}_{\mathrm{D}}$, instead of $I_{\mathrm{SU}-\mathrm{N}}$ which is a function of two variables $\tau$ and $\lambda$ and therefore not easy to analyze. The $\mathrm{SU}$ performance, in terms of $\tilde{I}_{\mathrm{SU}-\mathrm{N}}$, is studied in detail in Section 5

\subsection{The Constraint Threshold $P_{\mathrm{D}_{\mathrm{T}}}$ :}

We have already introduced $\tilde{I}_{\mathrm{SU}-\mathrm{N}}$, a function of $\tilde{P}_{\mathrm{D}}$, as the performance metric for SU. It is the maximum value of $I_{\mathrm{SU}-\mathrm{N}}$ over all such $\tau$ and $\lambda$ for which $P_{\mathrm{D}}=\tilde{P}_{\mathrm{D}}$. For the same detection probability, the corresponding value of $I_{\mathrm{PU}-\mathrm{N}}$ provides the measure of $\mathrm{PU}$ performance and is represented as $\tilde{I}_{\mathrm{PU}-\mathrm{N}}$, defined as follows

$$
\tilde{I}_{\mathrm{PU}-\mathrm{N}}=I_{\mathrm{PU}-\mathrm{N}} \text {, s.t. } P_{\mathrm{D}}=\tilde{P}_{\mathrm{D}} .
$$

Thus $\tilde{I}_{\mathrm{PU}-\mathrm{N}}$, a function of $\tilde{P}_{\mathrm{D}}$, is the performance metric for PU. Also, for all such $\tau$ and $\lambda$ for which $P_{\mathrm{D}}=\tilde{P}_{\mathrm{D}}, I_{\mathrm{PU}-\mathrm{N}}$ is a constant as detailed in Section 2 and 3 . Therefore we do not need to maximize it as we did for $\tilde{I}_{\mathrm{SU}-\mathrm{N}}$.

A decrease in detection probability always deteriorates the PU performance due to increase in interference by SU. Thus, $\tilde{I}_{\mathrm{PU}-\mathrm{N}}$ always decreases with decrease in $\tilde{P}_{\mathrm{D}}$. Therefore, for the optimization problem [12), at first, we select an appropriate detection probability threshold $P_{\mathrm{D}_{\mathrm{T}}}$ such that the PU performance $\tilde{I}_{\mathrm{PU}-\mathrm{N}}$ does not drop below a given threshold. For this, we define the $P U$ performance degradation (PPD) metric, for a certain value of $\tilde{I}_{\mathrm{PU}-\mathrm{N}}$, with respect to the value of $\tilde{I}_{\mathrm{PU}-\mathrm{N}}$ at $\tilde{P}_{\mathrm{D}}=1$. This metric is given as follows

$$
\Delta_{\mathrm{PU}}=\frac{\tilde{I}_{\mathrm{PU}-\mathrm{N}}\left(P_{\mathrm{D}}=1\right)-\tilde{I}_{\mathrm{PU}-\mathrm{N}}}{\tilde{I}_{\mathrm{PU}-\mathrm{N}}\left(P_{\mathrm{D}}=1\right)} .
$$

Thus, for some given maximum value of PU performance degradation factor say $\Delta_{\mathrm{T}}$, we can find the minimum eligible value of $\tilde{I}_{\mathrm{PU}-\mathrm{N}}$. We evaluate $P_{\mathrm{D}_{\mathrm{T}}}$ as the value of $\tilde{P}_{\mathrm{D}}$ corresponding to this minimum eligible $\mathrm{PU}$ rate. Therefore, we can write

$$
P_{\mathrm{D}_{\mathrm{T}}}=\tilde{P}_{\mathrm{D}} \text {, s.t. } \Delta_{\mathrm{PU}}=\Delta_{\mathrm{T}}
$$

In this way, we find an appropriate value for the constraint threshold $P_{\mathrm{D}_{\mathrm{T}}}$ that ensures the PU performance degradation remains within a given tolerable range. Later, for all $\tilde{P}_{\mathrm{D}}$ values greater than or equal to $P_{\mathrm{D}_{\mathrm{T}}}$, we maximize the $\mathrm{SU}$ performance $\tilde{I}_{\mathrm{SU}-\mathrm{N}}$. As a result, we eventually reach our goal of maximizing the SU performance for an acceptable degradation in PU performance, as 
summarized below.

$$
\begin{aligned}
& \max _{\tilde{P}_{\mathrm{D}}} \tilde{I}_{\mathrm{SU}-\mathrm{N}}, \text { s.t. } \tilde{P}_{\mathrm{D}} \geq P_{\mathrm{D}_{\mathrm{T}}}, \\
& \text { where, } \tilde{I}_{\mathrm{SU}-\mathrm{N}}=\max _{\tau, \lambda} I_{\mathrm{SU}-\mathrm{N}} \text {, s.t. } P_{\mathrm{D}}=\tilde{P}_{\mathrm{D}}, \\
& \text { and, } P_{\mathrm{D}_{\mathrm{T}}}=\tilde{P}_{\mathrm{D}} \text {, s.t. } \Delta_{\mathrm{PU}}=\Delta_{\mathrm{T}} .
\end{aligned}
$$

\section{Comparison with the Conventional Model}

To the best of the authors' knowledge the studies done so far on sensing-throughput trade-off have implicitly or explicitly made an important assumption that the SU-RX operates coherently with PU-TX. Under this assumption the received signal at SU-RX is given by

$$
Y_{\mathrm{SU}}= \begin{cases}X_{\mathrm{SU}}+W_{\mathrm{SU}} & \text { if } \hat{H}=0, H=0 . \\ X_{\mathrm{SU}}+W_{\mathrm{SU}}+X_{\mathrm{PU}}, & \text { if } \hat{H}=0, H=1\end{cases}
$$

While, the SU information rate for this model is given by

$$
\begin{aligned}
I_{\mathrm{SU}-\mathrm{N}} & =N_{c} I\left(X_{\mathrm{SU}} ; Y_{\mathrm{SU}} \mid \hat{H}, H\right) \\
& =(1-\tau) N \\
& \times\left[\operatorname{Pr}(\hat{H}=0, H=0) I\left(X_{\mathrm{SU}} ; Y_{\mathrm{SU}} \mid \hat{H}=0, H=0\right)\right. \\
& \left.+\operatorname{Pr}(\hat{H}=0, H=1) I\left(X_{\mathrm{SU}} ; Y_{\mathrm{SU}} \mid \hat{H}=0, H=1\right)\right] .
\end{aligned}
$$

For this model the capacity can be achieved if $X_{\mathrm{PU}}$ and $X_{\mathrm{SU}}$ are Gaussian distributed with probability density functions $(\mathrm{PDFs}) \mathcal{N}\left(0, \mathcal{P}_{\mathrm{PU}}\right)$ and $\mathcal{N}\left(0, \mathcal{P}_{\mathrm{SU}}\right)$ respectively, with $W_{\mathrm{PU}}$ and $W_{\mathrm{SU}}$ both are Gaussian distributed with $\operatorname{PDF} \mathcal{N}(0,1)$. Under these conditions, the achievable SU information becomes [6, 16, 17, 18, 19, 14, 13, 15],

$$
\begin{aligned}
I_{\mathrm{SU}-\mathrm{N}}=(1-\tau) N & \times\left[\pi_{0}\left(1-P_{\mathrm{F}}\right) \log \left(1+\mathcal{P}_{\mathrm{SU}}\right)\right. \\
& \left.+\pi_{1}\left(1-P_{\mathrm{D}}\right) \log \left(1+\frac{\mathcal{P}_{\mathrm{SU}}}{1+\mathcal{P}_{\mathrm{PU}}}\right)\right]
\end{aligned}
$$

In the conventional studies, optimal $\tau$ and $\lambda$ are found to maximize $I_{\mathrm{SU}-\mathrm{N}}$. Thus, the conventional optimization problem for the sensing-throughput trade-off is given by

$$
\max _{\tau, \lambda} I_{\mathrm{SU}-\mathrm{N}}, \text { s.t. } P_{\mathrm{D}} \geq P_{\mathrm{D}_{\mathrm{T}}}
$$
consequences on the sensing-throughput trade-off. Due to this assumption SU information rates for 
a single channel use, $I\left(X_{\mathrm{SU}} ; Y_{\mathrm{SU}} \mid \hat{H}=0, H=0\right)=\log \left(1+\mathcal{P}_{\mathrm{SU}}\right)$ and $I\left(X_{\mathrm{SU}} ; Y_{\mathrm{SU}} \mid \hat{H}=0, H=1\right)=$ $\log \left(1+\frac{\mathcal{P}_{\mathrm{SU}}}{1+\mathcal{P}_{\mathrm{PU}}}\right)$, are independent of $P_{\mathrm{D}}$. Now, since $P_{\mathrm{F}} \leq P_{\mathrm{D}}$ always holds, therefore according to 18 $I_{\mathrm{SU}-\mathrm{N}}$ is a decreasing function of $P_{\mathrm{D}}$ for a fixed $\tau$. Therefore, for the conventional model, $P_{\mathrm{D}} \geq P_{\mathrm{D}_{\mathrm{T}}}$ constraint is equivalent to $P_{\mathrm{D}}=P_{\mathrm{D}_{\mathrm{T}}}$. Hence, conventional model only requires maximizing $I_{\mathrm{SU}-\mathrm{N}}$ with respect to $\tau$ for an arbitrarily high value of $P_{\mathrm{D}_{\mathrm{T}}}$.

As a contrast, in our model, we assume that SU-RX is not coherent to PU-TX. Consequently, we have a mixture Gaussian channel, with a more complicated coupling relationship between the primary and the secondary users. Such coupling relationship is reflected not only by the presence or absence of an interference component in the received signals, which already showed up in conventional models, but also in a more complicated computation of the mutual information under our proposed mixture model, as explained in Section 3.2 This has two implications. Firstly, SU information rate $\hat{I}_{\mathrm{SU}-\mathrm{N}}$ for a single channel use depends on $P_{\mathrm{D}}$ for a fixed $\tau$ so that $P_{\mathrm{D}} \geq P_{\mathrm{D}_{\mathrm{T}}}$ constraint is not equivalent to $P_{\mathrm{D}}=P_{\mathrm{D}_{\mathrm{T}}}$ for our optimization problem. Thus we first maximize $I_{\mathrm{SU}-\mathrm{N}}$ with respect to $\tau$ for any given $P_{\mathrm{D}}=\tilde{P}_{\mathrm{D}}$ to seek a relationship over all. Secondly, the effect of $P_{\mathrm{D}}$ on $\mathrm{SU}$ performance depends on the interference caused by PU. The level of this interference depends on $\mathcal{P}_{\mathrm{PU}}$ for a fixed $\mathcal{P}_{\mathrm{SU}}$. Due to this, we have identified interference regimes for the SU performance based on $\mathcal{P}_{\mathrm{PU}}$ level, as discussed in Section 5.1. Also, in our analysis, instead of assigning an arbitrary value to the optimization problem constraint $P_{\mathrm{D}_{\mathrm{T}}}$ we assign a value based on the tolerable range of PU performance degradation as discussed in Section 3.2 .

\section{Results and Discussion}

This section discusses the relationship between the degrees-of-freedom (available to SU for communications) and the interference experienced by SU. This interference is caused by the PU signal due to imperfection in detection by SU and it affects the SU channel-information-rate. At the end of this section, we present and discuss the solution to the optimization problem under consideration.

As discussed in Sections 2 and 3 , the resulting interference channel for our system model is mixture Gaussian and in order to evaluate the information rates required for the analysis we need to compute the differential entropy of mixture Gaussian random variables. However, the differential entropy of a mixture Gaussian random variable does not have a closed form solution. Moreover, the optimization problem formulated in Section 3 involves quite a few parameters that are also non-linearly interdependent. Therefore, we have solved the optimization problem numerically. For the numerical evaluation of differential entropy of a mixture Gaussian random variable, we have implemented an algorithm based on the methodology proposed in [26]. In this section, we present the numerical results for $\pi_{1}=0.7, N=100$ and $\mathcal{P}_{\mathrm{SU}}=10$. Also, in Section 5.3 we present the 


\subsection{Objective function $\left(\tilde{I}_{S U-N}\right)$ versus $\tilde{P}_{\mathrm{D}}$}

The SU performance metric is $\tilde{I}_{\mathrm{SU}-\mathrm{N}}$ that is the maximum $I_{\mathrm{SU}-\mathrm{N}}$ at $P_{\mathrm{D}}=\tilde{P}_{\mathrm{D}} \cdot \tilde{I}_{\mathrm{SU}-\mathrm{N}}$ is thus a function of $\tilde{P}_{\mathrm{D}}$ as discussed in Section 3.2 . Here we discuss this SU performance metric as a function of $\tilde{P}_{\mathrm{D}}$ in detail in order to elaborate and explain the trade-off between the degrees of freedom available to $\mathrm{SU}$ for communications and the interference experienced by SU due to PU.

Degrees of freedom available to SU: For the SU system, the product $N_{\mathrm{c}} \psi_{1}$ that appears in (6) is the measure of the degrees of freedom (DOF) for communications. It quantifies the average number of channel-uses per frame used by SU for communications, in the long run, i.e., for infinitely large number of frames. Recall that we introduced the notions of 'channel-use' and 'frame' in Section 2 , where we described a synchronized frame structure for transmission of information in the system. The values of this DOF measure such that $P_{\mathrm{D}}=\tilde{P}_{\mathrm{D}}$ and the $\mathrm{SU}$ information rate is $\tilde{I}_{\mathrm{SU}-\mathrm{N}}$, are plotted against $\tilde{P}_{\mathrm{D}}$ in Figure 2. In this figure we present these numerical plots for different values of $\mathrm{PU}$ transmission power, $\mathcal{P}_{\mathrm{PU}}$. The figure shows that DOF available for $\mathrm{SU}$ decreases as the detection

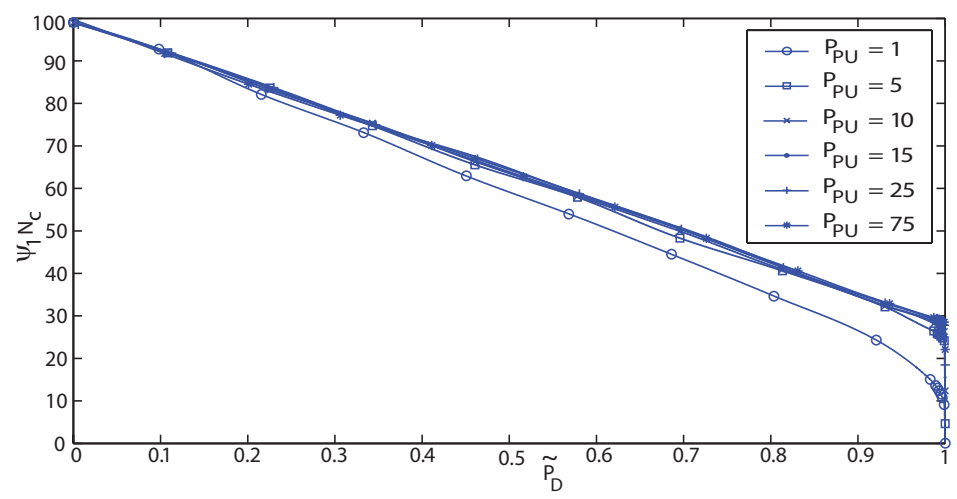

Figure 2: DOF available for SU for communications.

probability increases for all values of $\mathcal{P}_{\mathrm{PU}}$. This is because, increase in detection probability requires an increase in sensing time $\tau$ that results in a decrease in the communication period available for SU that is $N_{\mathrm{c}}=(1-\tau) N$. At the same time, the increase in $\tau$ also causes an increase in false alarm probability $P_{\mathrm{F}}$, which in turn causes an increase in the detection probability. Altogether, this decreases the probability that $\mathrm{SU}$ is $\mathrm{ON}$, i.e., $\psi_{1}=\operatorname{Pr}(\mathrm{SU}$ is on $)=\left(1-P_{\mathrm{F}}\right) \pi_{0}+\left(1-P_{\mathrm{D}}\right) \pi_{1}$. Hence, we conclude, that an increase in detection probability corresponds to a decrease in both $N_{\mathrm{c}}$ and $\psi_{1}$ thereby causing a decrease in DOF available for SU.

Interference caused by PU: In $(6), \hat{I}_{\mathrm{SU}-\mathrm{N}}$ is the mutual information of the SU system for a single channel use. It is the indicator for the interference caused by the PU system. The greater the interference, the lower will be the value of $\hat{I}_{\mathrm{SU}-\mathrm{N}}$. We represent the values of $\hat{I}_{\mathrm{SU}-\mathrm{N}}$, when $P_{\mathrm{D}}=\tilde{P}_{\mathrm{D}}$ and the $\mathrm{SU}$ information rate is $\tilde{I}_{\mathrm{SU}-\mathrm{N}}$, as $\tilde{\hat{I}}_{\mathrm{SU}-\mathrm{N}}$, i.e., with both tilde and a hat. $\tilde{\hat{I}}_{\mathrm{SU}-\mathrm{N}}$ is plotted against $\tilde{P}_{\mathrm{D}}$ in Figure 3 . In this figure we present these numerical plots for different values of $\mathrm{PU}$ transmission power, $\mathcal{P}_{\mathrm{PU}}$. The level of interference in the $\mathrm{SU}$ system caused by the $\mathrm{PU}$ is 


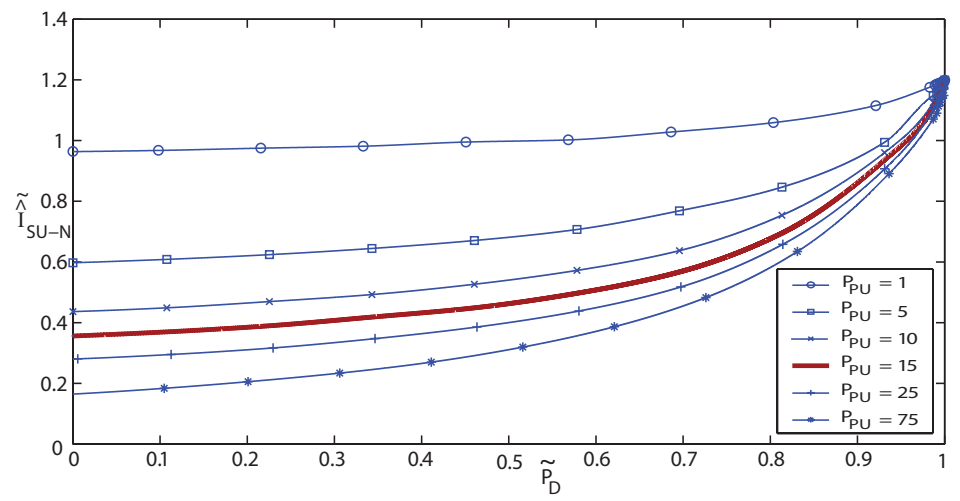

Figure 3: SU information for single channel-use vs $\tilde{P}_{\mathrm{D}}$.

determined by two factors, namely, the level of PU-TX transmission power $\mathcal{P}_{\mathrm{PU}}$ and the SU-TX detection quality that is characterized by the value of $\tilde{P}_{\mathrm{D}}$. Higher values of $\mathcal{P}_{\mathrm{PU}}$ and lower values of $\tilde{P}_{\mathrm{D}}$ result in high interference levels thereby resulting in lower values of $\tilde{\hat{I}}_{\mathrm{SU}-\mathrm{N}}$ in the SU system, and vice-versa. Thus, for a fixed $\mathcal{P}_{\mathrm{PU}}, \tilde{\hat{I}}_{\mathrm{SU}-\mathrm{N}}$ increases with increase in $\tilde{P}_{\mathrm{D}}$, as shown in Figure 3 . Also for a fixed $\tilde{P}_{\mathrm{D}} \neq 1, \tilde{\hat{I}}_{\mathrm{SU}-\mathrm{N}}$ is lower for higher values of $\mathcal{P}_{\mathrm{PU}}$, as shown in Figure 3 But, for $\tilde{P}_{\mathrm{D}}=1, \mathrm{PU}$ and SU do not interfere at all and both PU and SU channels are simply Gaussian. In that case, $\tilde{\hat{I}}_{\mathrm{SU}-\mathrm{N}}=(1 / 2) \log \left(1+\mathcal{P}_{\mathrm{SU}}\right)$ and is independent of $\mathcal{P}_{\mathrm{PU}}$. Therefore, all the curves meet at a single point at $\tilde{P}_{\mathrm{D}}=1$.

Degrees of freedom versus interference trade-off for SU: For SU system, on one hand the increase in detection probability causes a decrease in DOF available to $\mathrm{SU}$ for communications and on the other hand it causes an increase in the information rate for a single channel use. The overall resulting information rate for $\mathrm{SU}$, i.e., $\tilde{I}_{\mathrm{SU}-\mathrm{N}}$, is the product of DOF available and the information rate for a single channel use $\tilde{\hat{I}}_{\mathrm{SU}-\mathrm{N}}$. Hence, there exists a trade-off for the SU system between the available DOF and the interference level. This trade-off determines the overall trends in SU performance. The SU performance curves, for different values of $\mathcal{P}_{\mathrm{PU}}$, are shown in Figure 4. These curves are the numerical plots of $\tilde{I}_{S U-N}$ versus $\tilde{P}_{\mathrm{D}}$.

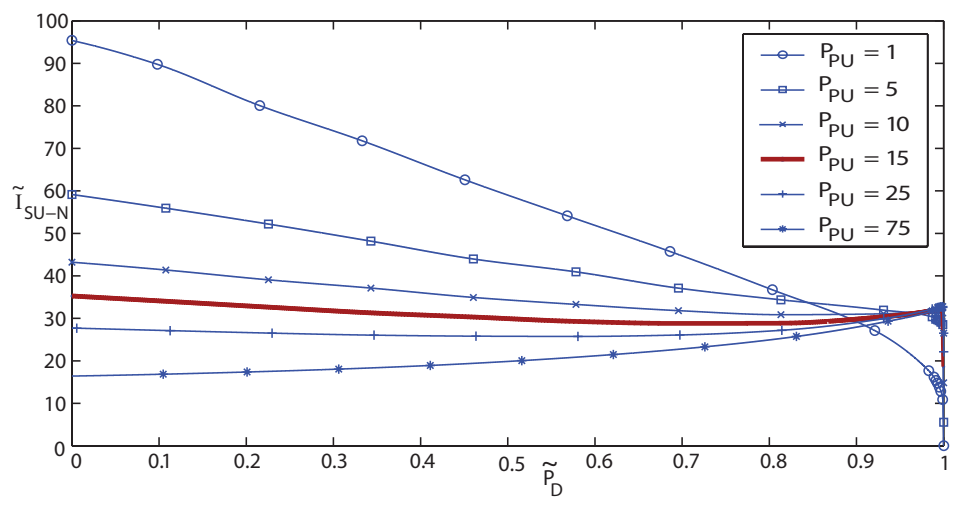

Figure 4: SU performance curves. 
Energy detector's inefficiency region: Near $\tilde{P}_{\mathrm{D}}=1$, a region of sharp decline in $\tilde{I}_{\mathrm{SU}-\mathrm{N}}$ exists, which we call as the energy detector's inefficiency (EDI) region. This sharp decline in SU

315 Near $\tilde{P}_{\mathrm{D}}=1$, a very high increase in $\tau$ is required for a very small increase in $\tilde{P}_{\mathrm{D}}$. The communication period $N_{\mathrm{c}}$ thus decreases quite significantly with $\tau$, thereby causing a sharp decrease in the degrees of freedom for communications as shown in Figure 2 During this EDI region, $\tilde{P}_{\mathrm{D}}$ is very high, i.e., nearly 1 . Therefore, in this high-detection-probability region, the interference caused by the PU 320 system is very negligible and does not affect $\tilde{\hat{I}}_{\mathrm{SU}-\mathrm{N}}$ much, no matter what the PU transmission power level is, as depicted in Figure 3. Hence, in this region the $\mathrm{SU}$ channel-rate $\tilde{\hat{I}}_{\mathrm{SU}-\mathrm{N}}$ is almost constant but the steep decline in DOF causes a similar decline in SU performance, as suggested by (6). For all selected values of $\mathcal{P}_{\mathrm{PU}}$, the EDI region starts almost at the same value of $\tilde{P}_{\mathrm{D}}$ defined as $P_{\mathrm{D}_{\mathrm{T} 2}}$. Numerical results, as presented in Figures 4 and 5 , show that if $\tilde{P}_{\mathrm{D}}$ exceeds $P_{\mathrm{D}_{\mathrm{T} 2}}$ and enters the EDI region, then it will not improve the PU performance much, but deteriorate the SU performance quite significantly, even when we are increasing the detection probability. This is what we mean by the word 'inefficiency' here. Thus, $\tilde{I}_{\mathrm{SU}-\mathrm{N}}\left(P_{\mathrm{D}_{\mathrm{T} 2}}\right)>>\tilde{I}_{\mathrm{SU}-\mathrm{N}}(1)$ while $\tilde{I}_{\mathrm{PU}-\mathrm{N}}\left(P_{\mathrm{D}_{\mathrm{T} 2}}\right) \approx \tilde{I}_{\mathrm{PU}-\mathrm{N}}(1)$. Thus, it is better to avoid the inefficient EDI region by sacrificing some $\tilde{P}_{\mathrm{D}}$ in order to have a better $\mathrm{SU}$ performance at almost no cost of PU performance. Hence, the appropriate value of $\tilde{P}_{\mathrm{D}}$ is upper of $P_{\mathrm{D}_{\mathrm{T} 2}}$ for some higher levels of $\mathrm{PU}$ transmission power as discussed in remark 1 in Section 5.3 .

Upper bound of SU performance for perfect detection: Consider the case of perfect detection, i.e., $P_{\mathrm{D}}=1$. For this case, $\mathrm{PU}$ and $\mathrm{SU}$ systems do not interfere with each other and both $\mathrm{PU}$ and $\mathrm{SU}$ channels are simply Gaussian. Therefore, at $P_{\mathrm{D}}=1$, the $\mathrm{SU}$ information rate detection, $\mathrm{SU}$ remains $\mathrm{ON}$ only when $\mathrm{PU}$ is $\mathrm{OFF}$ and there is no false alarm, i.e., the noise power level remains below the sensing threshold level $\lambda$. In this scenario, probability that $\mathrm{SU}$ is $\mathrm{ON}$ is thus given by $\psi_{1}\left(P_{\mathrm{D}}=1\right)=\left[1-\pi_{1}\right] \times\left[1-P_{\mathrm{F}}\right]$. Now consider a limiting case of infinitely high PU transmission power, to achieve perfect detection. In this case, SU-TX requires infinitesimally small sensing time, i.e., $\tau \approx 0$ to achieve perfect detection. Also due to very high PU transmission power, the false alarm probability can be kept infinitesimally small at the same time, by selecting a very large sensing threshold and thus $P_{\mathrm{F}} \approx 0$. For such a scenario, the upper bound on $I_{\mathrm{SU}-\mathrm{N}}$ for perfect detection can be achieved and is evaluated as $\nu_{1}=\frac{1}{2}\left(1-\pi_{1}\right) N \log \left(1+\mathcal{P}_{\mathrm{SU}}\right)$, using 6 .

PU transmission power threshold: As shown in Figure 4 , at $\tilde{P}_{\mathrm{D}}=1, \tilde{I}_{\mathrm{SU}-\mathrm{N}}$ increases with 345 increase in $\mathrm{PU}$ transmission power $\mathcal{P}_{\mathrm{PU}}$ but remains below $\nu_{1}$ since it is the upper bound on the $\mathrm{SU}$ information rate at $P_{\mathrm{D}}=1$. Now, consider the case of $\tilde{P}_{\mathrm{D}}=0$. On one hand, for higher values of PU transmission powers, $\tilde{I}_{\mathrm{SU}-\mathrm{N}}$ is less than $\nu_{1}$. On the other hand, for certain lower values of $\mathrm{PU}$ transmission powers, $\tilde{I}_{\mathrm{SU}-\mathrm{N}}\left(\tilde{P}_{\mathrm{D}}=0\right)$ is greater than $\nu_{1}$. This motivates us to categorize $\mathrm{SU}$ 
performance curves on the basis of SU performance in case of no detection, i.e., when $P_{\mathrm{D}}=0$. We

350 therefore define a threshold value of $\mathcal{P}_{\mathrm{PU}}$ represented as $\hat{\mathcal{P}}_{\mathrm{PU}}$ such that $\tilde{I}_{\mathrm{SU}-\mathrm{N}}\left(\tilde{P}_{\mathrm{D}}=0\right)=\nu_{1}$. We called the region of $\tilde{P}_{\mathrm{D}} \geq P_{\mathrm{D}_{\mathrm{T} 2}}$ as the EDI Region for SU. Thus the region of $\tilde{P}_{\mathrm{D}} \leq P_{\mathrm{D}_{\mathrm{T} 2}}$ is the Non-EDI Region for SU and consists of two regimes, namely the High and Low Interference Regimes for the SU. We define these regimes on the basis of PU transmission power level, as compared to the threshold $\hat{\mathcal{P}}_{\mathrm{PU}}$ that we have just introduced. These regimes are discussed in the subsequent discussion. We numerically estimate the value of $\hat{\mathcal{P}}_{\mathrm{PU}}$. For the system parameters that we have selected for our numerical study, $\hat{\mathcal{P}}_{\mathrm{PU}} \approx 15$. We call the corresponding SU performance curve as the Neutral $\tilde{I}_{\mathrm{SU}-\mathrm{N}}$ Curve. It shows negligible variations with respect to $\tilde{P}_{\mathrm{D}}$, indicating an underlying balance for the DOF and interference trade-off for SU, for the PU transmission-power level of $\hat{\mathcal{P}}_{\mathrm{PU}}$.

Interference regimes for SU (non-EDI region): The non-EDI region with a weak primary power, i.e., $\mathcal{P}_{\mathrm{PU}}<\hat{\mathcal{P}}_{\mathrm{PU}}$, is called the Low Interference Regime. The $\tilde{I}_{\mathrm{SU}-\mathrm{N}}$ versus $\tilde{P}_{\mathrm{D}}$ curves, above the Neutral $\tilde{I}_{\mathrm{SU}-\mathrm{N}}$ Curve, lie in the Low Interference Regime for SU. There are two different trends of SU performance in this regime. The first one is for the values of $\mathcal{P}_{\mathrm{PU}}$ close to $\hat{\mathcal{P}}_{\mathrm{PU}}$ in which $\tilde{I}_{\mathrm{SU}-\mathrm{N}}$ first decreases and then increases with increase in $\tilde{P}_{\mathrm{D}}$, for the non-EDI region as shown in Figure 4 . This trend constitutes the Stronger Low-Interference Regime. The second trend is for the lower values of $\mathcal{P}_{\mathrm{PU}}$ for which $\tilde{I}_{\mathrm{SU}-\mathrm{N}}$ decreases with increase in $\tilde{P}_{\mathrm{D}}$ for the non-EDI region as shown in Figure 4. This is because the effect of DOF dominates that of the interference in this regime of SU operation. As shown in Figure 2, for all values of $\mathcal{P}_{\mathrm{PU}}$, DOF decreases with increase in $\tilde{P}_{\mathrm{D}}$. Therefore in this Non-EDI region, $\tilde{I}_{\mathrm{SU}-\mathrm{N}}$ decreases with increase in $\tilde{P}_{\mathrm{D}}$. This trend constitutes the Weaker Low-Interference Regime. The non-EDI region with a stronger primary power, i.e., $\mathcal{P}_{\mathrm{PU}}>\hat{\mathcal{P}}_{\mathrm{PU}}$, is called the High Interference Regime. The effect of interference dominates that of the DOF in this regime of SU operation. As shown in Figure 3 , for all values of $\mathcal{P}_{\mathrm{PU}}, \tilde{\hat{I}}_{\mathrm{SU}-\mathrm{N}}$ increases with increase in $\tilde{P}_{\mathrm{D}}$, because of the decrease in interference within the SU system due to PU. Therefore in this Non-EDI region, $\tilde{I}_{\mathrm{SU}-\mathrm{N}}$ increases with increase in $\tilde{P}_{\mathrm{D}}$. The $\tilde{I}_{\mathrm{SU}-\mathrm{N}}$ versus $\tilde{P}_{\mathrm{D}}$ curves, below the Neutral $\tilde{I}_{\mathrm{SU}-\mathrm{N}}$ Curve, lie in the High Interference Regime for SU. Note that such interesting trends model and can only be observed under the mixture Gaussian model proposed in our paper.

\section{2. $\tilde{I}_{P U-N}$ versus $\tilde{P}_{\mathrm{D}}$}

The $\tilde{I}_{P U-N}$ versus $\tilde{P}_{\mathrm{D}}$ curves are the PU performance curves. Figure 5 shows that $\tilde{I}_{P U-N}$ increases with $\tilde{P}_{\mathrm{D}}$ for all values of $\mathcal{P}_{\mathrm{PU}}$. The trends in these curves are because of the interference in the PU system due to SU. The effect of this interference in PU system depends on the level of $\mathcal{P}_{\mathrm{PU}}$ and the value of $\tilde{P}_{\mathrm{D}}$. For a given $\mathcal{P}_{\mathrm{PU}}$, an increase in $\tilde{P}_{\mathrm{D}}$ causes a decrease in interference from SU and thus PU performance increases. On the other hand, for a given $\tilde{P}_{\mathrm{D}}$, increase in $\mathcal{P}_{\mathrm{PU}}$ increases the $\mathrm{PU}$ information rate thereby increasing the $\mathrm{PU}$ performance. 


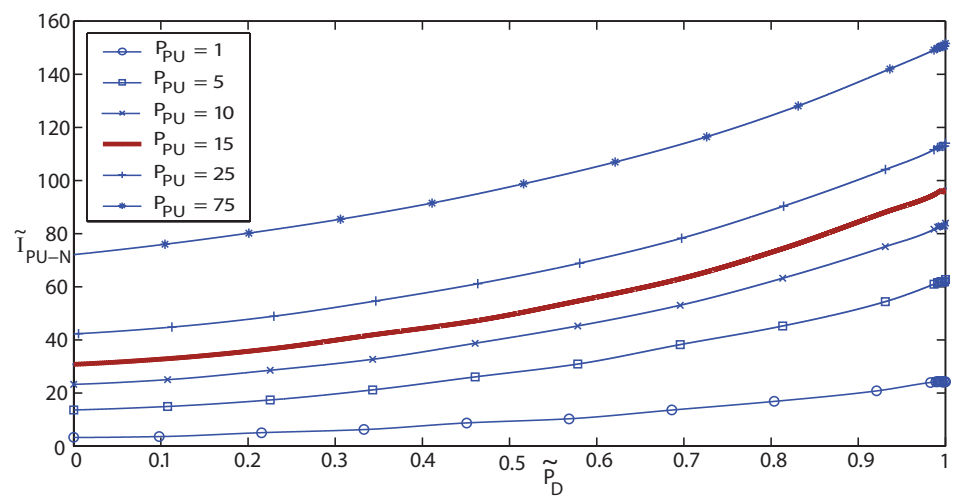

Figure 5: PU performance curves.

\subsection{Optimal Solution}

For quite a few selected values of $\mathcal{P}_{\mathrm{PU}}$ the results for $\Delta_{\mathrm{T}}=0.04$ are given in Table 2 and 3 , Here in these tables, $\tilde{P}_{\mathrm{D} 0}$ and $\tilde{\Delta}_{0}$ are the values of detection probability and $\Delta_{\mathrm{PU}}$, respectively, for the corresponding optimal solution. Following are some remarks related to the numerical solutions presented in Table 2 and 3 .

\begin{tabular}{ccccccc}
\multicolumn{2}{c}{ Table 2: Optimized Solutions for } & \multicolumn{4}{c}{$\Delta_{\mathrm{T}}=0.04}$, & \multicolumn{2}{c}{$\mathcal{P}_{\mathrm{SU}}=10$, Energy Detector } \\
$\mathcal{P}_{\mathrm{PU}}$ & $\max I_{\mathrm{SU}}$ & $\tilde{\tau}_{0}$ & $\tilde{\lambda}_{0}$ & $\tilde{P}_{\mathrm{D} 0}$ & $\tilde{P}_{\mathrm{F} 0}$ & $\tilde{\Delta}_{0}$ \\
\hline 1 & 20.4258 & 0.3402 & 40.9927 & 0.9687 & 0.2017 & 0.04 \\
5 & 30.6721 & 0.1216 & 24.9403 & 0.9812 & 0.0165 & 0.04 \\
10 & 31.7490 & 0.0900 & 21.9999 & 0.9910 & 0.0089 & 0.0176 \\
$\hat{\mathcal{P}}_{\mathrm{PU}} \approx 15$ & 32.0381 & 0.0800 & 21.0000 & 0.9950 & 0.0071 & 0.0092 \\
25 & 32.6405 & 0.0700 & 20.9996 & 0.9970 & 0.0038 & 0.0105 \\
75 & 32.7804 & 0.0500 & 19.0121 & 0.9989 & 0.0019 & 0.0067 \\
\hline
\end{tabular}

Table 3: Optimized Solutions for $\Delta_{\mathrm{T}}=0.04, \mathcal{P}_{\mathrm{SU}}=0.1$, Energy Detector

\begin{tabular}{ccccccc}
$\mathcal{P}_{\mathrm{PU}}$ & $\max I_{\mathrm{SU}}$ & $\tilde{\tau}_{0}$ & $\tilde{\lambda}_{0}$ & $\tilde{P}_{\mathrm{D} 0}$ & $\tilde{P}_{\mathrm{F} 0}$ & $\tilde{\Delta}_{0}$ \\
\hline 0.1 & 2.0473 & 0.0938 & 9.1668 & 0.5167 & 0.4472 & 0.04 \\
2 & 1.7302 & 0.0257 & 9.5706 & 0.2925 & 0.0153 & 0.04 \\
$\hat{\mathcal{P}}_{\mathrm{PU}} \approx 4.1$ & 1.3386 & 0.0114 & 13.2715 & 0.1350 & 0.00036 & 0.04 \\
8 & 1.2250 & 0.1100 & 23.0000 & 0.9950 & 0.0177 & 0.00066 \\
16 & 1.2820 & 0.1000 & 26.000 & 0.999 & 0.0037 & 0.000035 \\
\hline
\end{tabular}

1. When $\mathcal{P}_{\mathrm{PU}}>\hat{\mathcal{P}}_{\mathrm{PU}}$, the $\mathrm{SU}$ performance curve is in the High Interference Regime for SU where $\tilde{I}_{\mathrm{SU}-\mathrm{N}}$ increases with increase in $\tilde{P}_{\mathrm{D}}$ for the non-EDI region as shown in Figure 4 . In this case, maximum value of $\tilde{I}_{\mathrm{SU}-\mathrm{N}}$ is at the maximum allowed value of $\tilde{P}_{\mathrm{D}}$, i.e., $P_{\mathrm{D}_{\mathrm{T} 2}}$. We cannot have a better detection probability, because, beyond $\tilde{P}_{\mathrm{D}}=P_{\mathrm{D}_{\mathrm{T} 2}}$ the EDI region begins, and in this region the SU performance decreases with increase in detection probability, as discussed 
in Section 5.1. Thus we conclude that, for a practical system where the PU transmissionpower level is very high, the SU's signal detector should be designed for the maximum allowed detection probability in order to avoid any chance of interference from PU.

2. Note that for the High Interference Regime for SU, our algorithm gives us the value of detection probability at which the EDI region begins, i.e., $P_{\mathrm{D}_{\mathrm{T} 2}}$, since it is the detection probability corresponding to the optimal solution for this regime, according to remark 1 .

3. When $\mathcal{P}_{\mathrm{PU}}<\hat{\mathcal{P}}_{\mathrm{PU}}$, the SU performance curve is in the Low Interference Regime for SU. For this regime, the interference caused by $\mathrm{PU}$ is so low that the contention occurring due to the decrease in detection probability does not deteriorate SU performance much. Rather, it causes a decrease in sensing time which implies an increase in degrees of freedom for communications. Consequently, this results in an increase in SU information rate with decrease in detection probability. Below a certain detection probability, the SU performance for the weaker low interference regime even exceeds $\nu_{1}$, i.e., the upper bound on SU performance for the high interference regime. Thus, for the Weaker Low Interference Regime $\tilde{I}_{\mathrm{SU}-\mathrm{N}}$ decreases with increase in $\tilde{P}_{\mathrm{D}}$ for the non-EDI region as shown in Figure 4 . Therefore in this regime, the maximum value of $\tilde{I}_{\mathrm{SU}-\mathrm{N}}$ is at the minimum allowed $\tilde{P}_{\mathrm{D}}$, i.e., $P_{\mathrm{D}_{\mathrm{T}}}$. We cannot have a further lower detection probability, because, by definition $(15), P_{\mathrm{D}_{\mathrm{T}}}$ is the value of detection probability at which the PU performance degradation factor $\Delta_{\mathrm{PU}}$ is at its maximum allowed value $\Delta_{\mathrm{T}}$. Further decreasing the detection probability increases $\Delta_{\mathrm{PU}}$ which violates our optimality constraint.

4. According to remark 3 , the value of PU performance degradation factor corresponding to the optimal solution for the Weaker Low Interference Regime is equal the maximum allowed value $\Delta_{\mathrm{T}}$. Thus, $\tilde{\Delta}_{0}=\Delta_{\mathrm{T}}$ for the Weaker Low Interference Regime, as indicated by the solutions presented in Table 2 and 3 . Therefore we can also infer that, for a practical system, the tolerance-level for the PU performance-degradation can be exploited to increase the SU performance by sacrificing $P_{\mathrm{D}}$ but only for the Weaker Low Interference Regime of $S U$.

5. Table 2 shows that for $\mathrm{SU}$ transmission power, $\mathcal{P}_{\mathrm{SU}}=10$, the optimal $\mathrm{SU}$ information rate is quite high in case of high $\mathrm{PU}$ transmission power provided the detection probability is very high. Thus for the assumed values of the system parameters we can conclude that if $4 \%$ performance degradation can be tolerated by PU then in case of strong SU transmission power level, e.g., 10, SU has the best optimal-performance in case of High Interference Regime provided the detection probability is very high. This is because for high SU transmission power the detection probability should be as much high as possible, i.e., nearly 1 , in order to avoid a high interference in PU system. Also, for very high detection probability, high 
PU transmission power ensures smaller sensing time for SU thereby causing high degrees of freedom for communications and hence high information rate for SU.

6. Table 3 shows that for a weak SU transmission power, i.e., $\mathcal{P}_{\mathrm{SU}}=0.1$, the optimal SU information rate is quite high in case of low $\mathrm{PU}$ transmission power. This is because, a low SU transmission power level causes a low interference to the PU system. In this case PU can tolerate a large extent of contention. This allows SU to operate at such low detection probability levels such that the sensing time is small which implies high degrees-of-freedom for communications for SU. This results in a higher SU performance for the weaker low interference regime which is even higher than the upper bound on SU performance for high interference regime.

7. According to remarks 5 and 6, for a practical system, the optimal secondary user throughput is high when both SU and PU transmission-power levels are either low or high.

\subsection{Comparison with Conventional Studies}

Recall from Section 4 that in the conventional studies, a major consequence of the assumption that SU-RX is coherent to PU-TX is that the inequality constraint in $(9)$, on the detection probability, is always equivalent to the equality constraint, regardless of the interference caused by PU to SU. However, as discussed in Section 5.3, our results demonstrate that under the assumption that SU-RX is incoherent to PU-TX, the SU system can improve its throughput by sacrificing the detection probability and maintaining only the minimum required detection quality, but for the low interference regime. Thus, under the incoherent assumption, the inequality constraint on the detection probability is equivalent to the equality constraint only for this regime. Note that this does not hold for the high interference regime where the detection probability cannot be sacrificed, as discussed in Section 5.3 .

\section{Nearest Neighbor Decoding for PU}

In the case of a Gaussian channel, the capacity, i.e., $1 / 2 \log (1+\mathrm{SNR})$ can be achieved for Gaussian channel input and the nearest neighbor decoding (NND) is the optimal decoding rule for the receiver [27]. Since the PU system does not know about the existence of the SU system therefore we can assume that it anticipates the channel as Gaussian. For this, PU-TX uses Gaussian channelinput and NND is the decoding rule for PU-RX. For an ergodic non-Gaussian channel, the achievable channel capacity is $1 / 2 \log (1+\mathrm{SNR})$ if the receiver uses NND [25]. Also, this capacity is achieved for the Gaussian channel-input [25]. Therefore, in our new scenario for PU in which PU-TX still uses the Gaussian channel-input but PU-RX uses the nearest neighbor decoding rather than the

$\mathrm{ML}$, the mutual information for a single channel use is $\hat{I}_{\mathrm{PU}-\mathrm{Nc}}=1 / 2 \log \left[1+\left(\mathcal{P}_{\mathrm{PU}} / \sigma_{\mathrm{Z} U}^{2}\right)\right]$ during the communication period of SU, where $\sigma_{\mathrm{Z}_{\mathrm{PU}}}^{2}=1+\left(1-P_{\mathrm{D}}\right) \mathcal{P}_{\mathrm{SU}}$. The mutual information for 
a single channel use during the sensing period of SU, $\hat{I}_{\mathrm{PU}-\mathrm{Ns}}$, is the same as before. Therefore, the mutual information for the PU system over $N$ channel uses can be evaluated as follows by using (3).

$$
\begin{aligned}
I_{\mathrm{PU}-\mathrm{N}} & =\pi_{1} N \frac{\tau}{2} \log \left(1+\mathcal{P}_{\mathrm{PU}}\right) \\
& +\pi_{1} N \frac{(1-\tau)}{2} \log \left(1+\frac{\mathcal{P}_{\mathrm{PU}}}{1+\left(1-P_{\mathrm{D}}\right) \mathcal{P}_{\mathrm{SU}}}\right) .
\end{aligned}
$$

Thus, when PU-RX employs NND, only the expression for $I_{\mathrm{PU}-\mathrm{N}}$ changes. The rest of the details remains the same as that in case of ML-detector for PU-RX. We can find the new optimal sensing time and threshold for SU-TX by using the updated expression for $I_{\mathrm{PU}-\mathrm{N}}$ given by $(20)$ and adopting the same methodology discussed earlier.

\subsection{Effect of Fading}

In this section, we discuss a simple scenario that can give us some idea how fading can affect the degrees of freedom and interference trade-off. However, a more thorough analysis with fading needs a dedicated study and is left for future work. For this study, we assume that PU-RX uses NND decoding and fading only occurs over the SU-TX to PU-RX channel. Let $\Phi_{\mathrm{SP}}$ be a random variable representing the fading coefficient over this channel, such that the fading deteriorates the $\mathrm{SU}$ interference to PU, i.e., $\theta_{\mathrm{SP}}=\mathrm{E}\left[\left|\Phi_{\mathrm{SP}}\right|^{2}\right] \in(0,1)$. Note that $\mathrm{E}[$.$] represents the expected value$ of the random variable. The $\mathrm{PU}$ received signal for this scenario will be $Y_{\mathrm{PU}}=X_{\mathrm{PU}}+Z_{\mathrm{PU}}$, where the noise at the $\mathrm{PU}$ receiver is $Z_{\mathrm{PU}}=W_{\mathrm{PU}}+\alpha \Phi_{\mathrm{SP}} X_{\mathrm{SU}}$. Similar to 20, the $\mathrm{PU}$ information rate under the stated fading scenario and NND decoding is given by, 25,

$$
\begin{aligned}
I_{\mathrm{PU}-\mathrm{N}} & =\pi_{1} N \frac{\tau}{2} \log \left(1+\mathcal{P}_{\mathrm{PU}}\right) \\
& +\pi_{1} N \frac{(1-\tau)}{2} \log \left(1+\frac{\mathcal{P}_{\mathrm{PU}}}{1+\left(1-P_{\mathrm{D}}\right) \theta_{\mathrm{SP}} \mathcal{P}_{\mathrm{SU}}}\right) .
\end{aligned}
$$

Note that here we assume that $\Phi_{\mathrm{SP}}$ is independent of all other random variables. Since $0<\theta_{\mathrm{SP}}<1$, therefore, comparing (20) and 21) reveals that the PU performance improves under the fading over SU-TX to PU-RX channel that deteriorates the SU interference to PU. Therefore, for a fixed minimum allowed degradation in PU performance, PU can tolerate more interference from SU under such fading scenario. Thus, SU can further improve its performance under such scenario by sacrificing the detection probability further. However, as discussed in Section 5.3, such improvement in SU performance is only possible under the low interference regime.

\section{Energy Detector versus Envelope Detector}

Earlier we assumed that SU-TX uses the optimal signal detector under the Neyman-Pearson rule [28, i.e., the energy detector in our system model under the Gaussian assumption. In this 
section, we numerically solve the same optimization problem for a relatively less sensitive envelope detector. For the envelope detector, instead of evaluating the closed form expressions for $P_{\mathrm{D}}$ and $P_{\mathrm{F}}$, we adopt the Monte Carlo simulation approach. The SU and PU performances versus the detection probability for both the detectors are presented in Figures 6 and 7 respectively. A particular optimal solution for the envelope detector is presented in Table 4

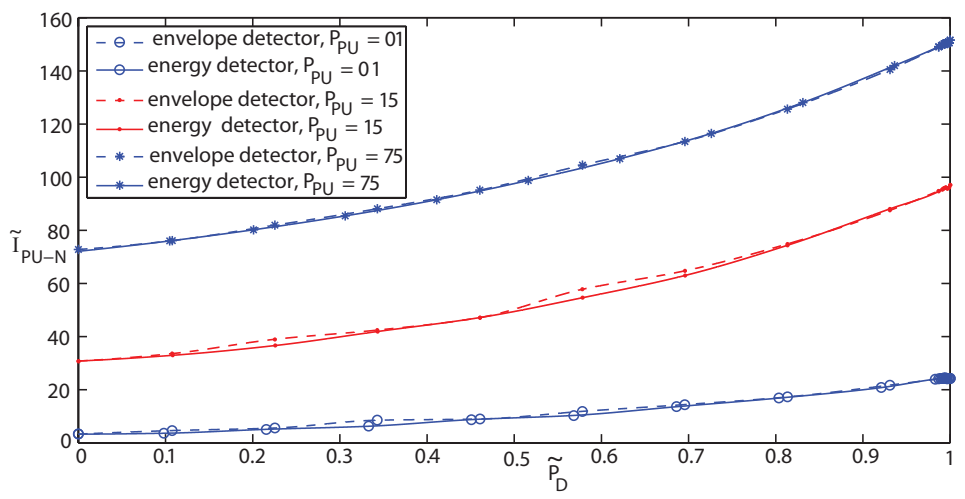

Figure 6: PU performance curves for envelope and energy detectors.

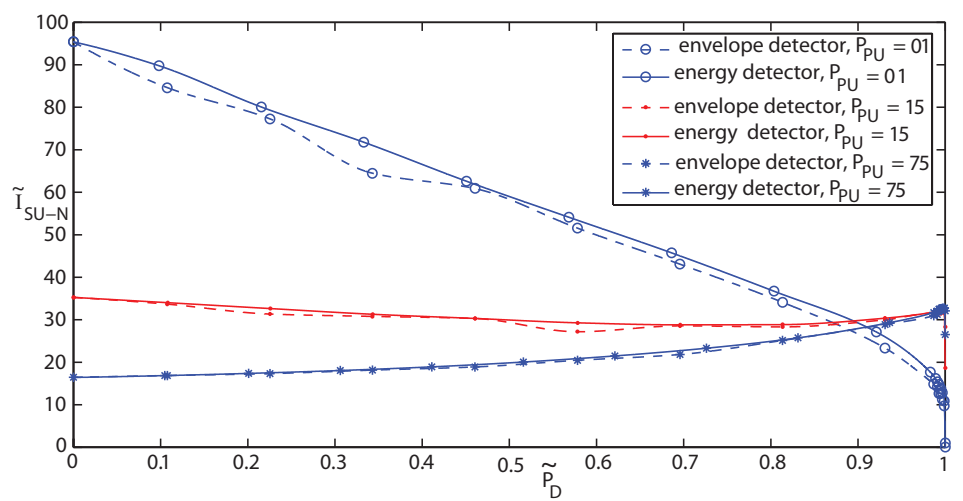

Figure 7: SU performance curves for envelope and energy detectors.

As compared to Table 2. Table 4 shows that the optimal sensing time is almost the same as in case of the optimal (energy) detector and the most significant effect of using the envelope detector instead of the energy detector is a considerable reduction in the value of optimal sensing threshold ${ }_{475} \tilde{\lambda}_{0}$. This is because the envelope detector is less sensitive to the PU signal than the energy detector. To compensate this decrease in sensitivity, the sensing threshold needs to be lowered while keeping the sensing time the same as in case of the optimal (energy) detector. Also, this reduction in the sensing threshold increases the false alarm probability for the envelope detector as compared to the energy detector while the sensing time remains the same.

Figure 6 shows that there is no significant difference in PU performances for both the detectors. On the other hand, Figure 7 shows that in case of the high interference regime there is almost no difference in SU performances for both the detectors but in case of the low interference regime 


\begin{tabular}{ccccccc}
\multicolumn{2}{c}{ Table 4: Optimized Solutions for $\Delta_{\mathrm{T}}=0.04, \mathcal{P}_{\mathrm{SU}}=10$, Envelope Detector } \\
$\mathcal{P}_{\mathrm{PU}}$ & $\max I_{\mathrm{SU}}$ & $\tilde{\tau}_{0}$ & $\tilde{\lambda}_{0}$ & $\tilde{P}_{\mathrm{D} 0}$ & $\tilde{P}_{\mathrm{F} 0}$ & $\tilde{\Delta}_{0}$ \\
\hline 1 & 17.8873 & 0.3059 & 21.3106 & 0.9687 & 0.3441 & 0.0398 \\
$\hat{\mathcal{P}}_{\mathrm{PU}} \approx 15$ & 31.8157 & 0.0800 & 11.0000 & 0.9930 & 0.0073 & 0.0135 \\
75 & 32.6701 & 0.0500 & 8.0000 & 0.9990 & 0.0047 & 0.0068 \\
\hline
\end{tabular}

$\mathrm{SU}$ performance for the envelope detector is slightly less than that for the energy detector. This is because in the high interference regime PU transmission power is very high, and together with

485

the appropriate decrease in the sensing threshold, it almost completely compensates the decrease in detector's sensitivity.

Frequency bands dedicated for TV-broadcasting are the potential spectral resource that can be used by the intelligent wireless devices (SUs) that can sense the presence or absence of the TV signal in the frequency band of interest and access the channel if it is found idle [29. Since TV broadcasting is done at a high transmission power level therefore, according to our results in this section, even if the signal detector of the SU wireless device performs suboptimally SU can still achieve the optimal-detector's performance level just by adjusting the sensing parameters, e.g., in the same way as the sensing threshold for the envelope detector is tuned to achieve the optimal detector's performance level in the high interference regime, in our system model.

\section{Conclusion}

In this paper, we explored the degrees-of-freedom and interference trade-off for a simple cognitive radio system under an assumption that the secondary-user (SU) receiver operates incoherently with the primary-user $(\mathrm{PU})$ transmitter. This assumption makes our system model practically more rigorous, as compared to the conventional studies where a coherent operation is assumed. Due to the incoherent operation of SU receiver in our model, the resulting interference channel is mixtureGaussian. Our objective is to find the optimal sensing threshold and sensing time for the signal detector that is used by the SU to detect the channel occupancy by the PU. The optimality criterion is to maximize the $\mathrm{SU}$ performance and at the same time keep the PU performance degradation, caused by the interference due to SU, within a tolerable range. We numerically solve this optimization design-problem since the closed form solution is not possible. As a further novel contribution, we define the interference regimes for SU performances on the basis of PU transmission power level. We also find that the tolerance level for PU performance degradation can be exploited to increase the SU performance but only for the Weaker Low Interference Regime for SU. The effect of a more robust decoding strategy, namely, nearest neighbor decoding approach, at PU-RX is also elaborated. Finally, we demonstrate that even if the signal detector of SU performs suboptimally, SU can still achieve the optimal detector's performance level in the high interference regime just by adjusting the sensing parameters accordingly. 


\section{Acknowledgements}

This work is supported in part by the Board of Regents of Louisiana under contract LEQSF(2009tract CNS-1018273.

\section{References}

[1] D. Čabrić, S. M. Mishra, D. Willkomm, R. Brodersen, A. Wolisz, A cognitive radio approach for usage of virtual unlicensed spectrum, in: Proc. of 14th IST Mobile Wireless Communications Summit, 2005, pp. 1-4.

[2] J. Mitola, Cognitive radio for flexible mobile multimedia communications, in: IEEE International Workshop on Mobile Multimedia Communications, San Diego, CA, USA, 1999, pp. 3 10.

[3] E. Tragos, S. Zeadally, A. Fragkiadakis, V. Siris, Spectrum assignment in cognitive radio networks: A comprehensive survey, IEEE Communications Surveys Tutorials 15 (3) (2013) 11081135 .

[4] E. Biglieri, J. Proakis, S. Shamai, Fading channels: Information-theoretic and communications aspects, IEEE Transactions on Information Theory 44 (6) (1998) 2619-2692.

[5] Y. Polyanskiy, H. V. Poor, S. Verdú, Dispersion of the Gilbert-Elliott channel, IEEE Transactions on Information Theory 57 (4) (2011) 1829-1848.

[6] Y.-C. Liang, Y. Zeng, E. C. Y. Peh, A. T. Hoang, Sensing-throughput tradeoff for cognitive radio networks, IEEE Transactions on Wireless Communications 7 (4) (2008) 1326-1337.

[7] H. Pradhan, S. Kalamkar, A. Banerjee, Sensing-throughput tradeoff in cognitive radio with random arrivals and departures of multiple primary users, IEEE Communications Letters 19 (3) (2015) 415-418.

[8] E. Bedeer, O. Dobre, M. Ahmed, K. Baddour, Rate-interference tradeoff in ofdm-based cognitive radio systems, IEEE Transactions on Vehicular Technology PP (99) (2014) 1-7.

[9] A. Kortun, T. Ratnarajah, M. Sellathurai, Y.-C. Liang, Y. Zeng, On the eigenvalue-based spectrum sensing and secondary user throughput, IEEE Transactions on Vehicular Technology 63 (3) (2014) 1480-1486.

[10] S. Zhang, H. Zhao, S. Wang, J. Wei, A cross-layer rethink on the sensing-throughput tradeoff for cognitive radio networks, IEEE Communications Letters 18 (7) (2014) 1226-1229. 
[11] S. K. Sharma, S. Chatzinotas, B. Ottersten, A hybrid cognitive transceiver architecture: Sensing-throughput tradeoff, in: International Conference on Cognitive Radio Oriented Wireless Networks and Communications (CROWNCOM), IEEE, 2014, pp. 143-149.

[12] M.-F. Hsu, T.-Y. Wang, C.-T. Yu, A unified spectrum sensing and throughput analysis model in cognitive radio networks, Wireless Communications and Mobile Computing 15 (9) (2015) $1368-1375$.

[13] S. Zarrin, T. J. Lim, Throughput-sensing tradeoff of cognitive radio networks based on quickest sensing, in: IEEE International Conference on Communications (ICC), Kyoto, Japan, 2011, pp. $1-5$.

[14] S. Stotas, A. Nallanathan, Overcoming the sensing-throughput tradeoff in cognitive radio networks, in: IEEE International Conference on Communications (ICC), Cape Town, South Africa, 2010, pp. $1-5$.

[15] L. Tang, Y. Chen, E. L. Hines, M.-S. Alouini, Effect of primary user traffic on sensingthroughput tradeoff for cognitive radios, IEEE Transactions on Wireless Communications 10 (4) (2011) 1063-1068.

[16] Y.-J. Choi, Y. Xing, S. Rangarajan, Overhead-throughput tradeoff in cooperative cognitive radio networks, in: IEEE Wireless Communications and Networking Conference (WCNC), Budapest, Hungary, 2009, pp. 1-6.

[17] S. Huang, X. Liu, Z. Ding, Optimal-sensing transmission structure for dynamic spectrum access, in: IEEE INFOCOM, Rio de Janeiro, Brazil, 2009, pp. 2295 - 2303.

[18] Y. Pei, Y.-C. Liang, K. C. Teh, K. H. Li, Sensing-throughput tradeoff for cognitive radio networks: A multiple-channel scenario, in: IEEE International Symposium on Personal, Indoor and Mobile Radio Communications (PIMRC), Tokyo, Japan, 2009, pp. 1257 - 1261.

[19] E. C. Y. Peh, Y.-C. Liang, Y. L. Guan, Y. Zeng, Optimization of cooperative sensing in cognitive radio networks: A sensing-throughput tradeoff view, IEEE Transactions on Vehicular Technology 58 (9) (2009) 5294-5299.

[20] S. Wei, V. Chakravarthy, Z. Wu, R. Kannan, Sensing and transmission in probabilistically 570 interference limited cognitive radio systems, in: IEEE Global Telecommunications Conference (GLOBECOM), Houston, TX, USA, 2011, pp. $1-5$.

[21] A.-A. Ali, S. Wei, Degrees-of-freedom and interference tradeoff for a mix-gaussian cognitive radio channel, in: IEEE Annual Conference on Information Sciences and Systems (CISS), Baltimore, MD, USA, 2013, pp. 1-6. 
[22] H. Urkowitz, Energy detection of unknown deterministic signals, Proceedings of the IEEE 55 (4) (1967) 523-531.

[23] F. F. Digham, M.-S. Alouini, M. K. Simon, On the energy detection of unknown signals over fading channels, IEEE Transactions on Communications 55 (1) (2007) 21-24.

[24] I. S. Gradshteyn, I. M. Ryzhik, Table of Integrals, Series and Products, pp. 892, 899, Elsevier, 2007.

[25] A. Lapidoth, Nearest neighbor decoding for additive non-gaussian noise channels, IEEE Transactions on Information Theory 42 (5) (1996) 1520-1529.

[26] M. F. Huber, T. Bailey, H. Durrant-Whyte, U. D. Hanebeck, On entropy approximation for gaussian mixture random vectors, in: IEEE International Conference on Multisensor Fusion and Integration for Intelligent Systems, MFI 2008, Seoul, 2008, pp. 181-188.

[27] T. M. Cover, J. A. Thomas, Elements of Information Theory, pp. 264, John Wiley and Sons, 2006.

[28] H. L. V. Trees, Detection, Estimation, and Modulation Theory - Vol 1, pp. 33, John Wiley and Sons, 2001.

590

[29] C. Cordeiro, K. Challapali, D. Birru, N. Sai Shankar, IEEE 802.22: the first worldwide wireless standard based on cognitive radios, in: First IEEE International Symposium on New Frontiers in Dynamic Spectrum Access Networks (DySPAN), 2005, pp. 328-337. 
Ahsan-Abbas Ali got his BSc in Electrical Engineering from University of Engineering and Technology (UET), Lahore, Pakistan in 2007. From 2007 to 2009, he worked as a Research Associate at Networks and Communications Lab at Lahore University of Management Sciences, Pakistan. He served as a Lecturer/Lab-Engineer at UET Lahore from 2008 to 2010. He is currently a PhD student at the school of EECS at Louisiana State University (LSU), Baton Rouge. He has been awarded with LSU Graduate Tuition Award 2011 and ECE James R. Lewis Scholarship 2014. His interests include modeling and analysis of communication systems, and data mining. 
Dr. Shuangqing Wei got his B.E and M.E in Electrical Engineering from Tsinghua University in 1995 and 1998, respectively. He started his academic career at Louisiana State University after obtaining his Ph.D. from the University of Massachusetts, Amherst in 2003. He is currently a Tenured Associate Professor in the Division of ECE of the School of EECS at LSU. His research interests include information theory, statistical inference, communication theory and their applications in the areas of telecommunication networks. 


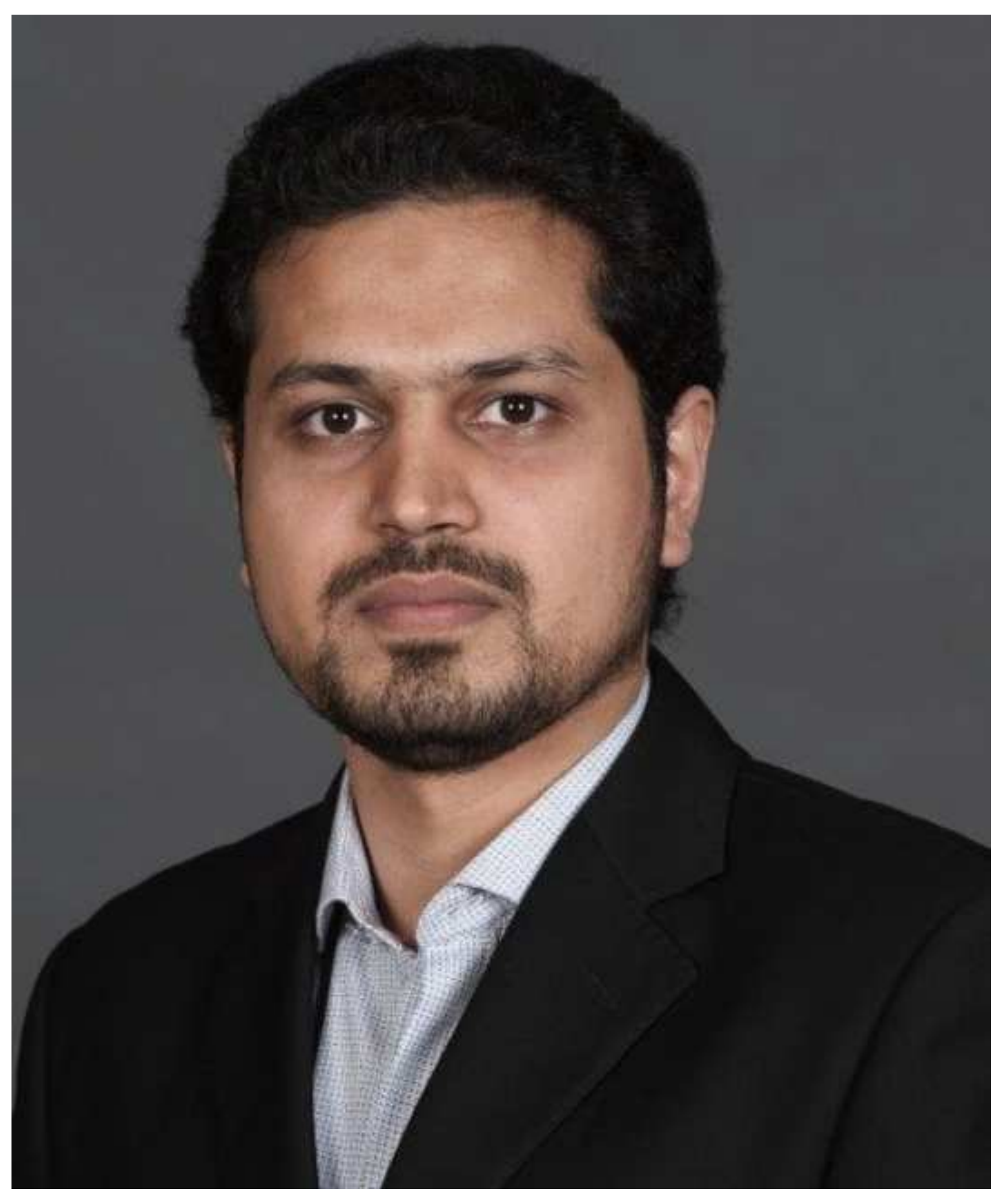

\section{Ahsan-Abbas All's Photo}




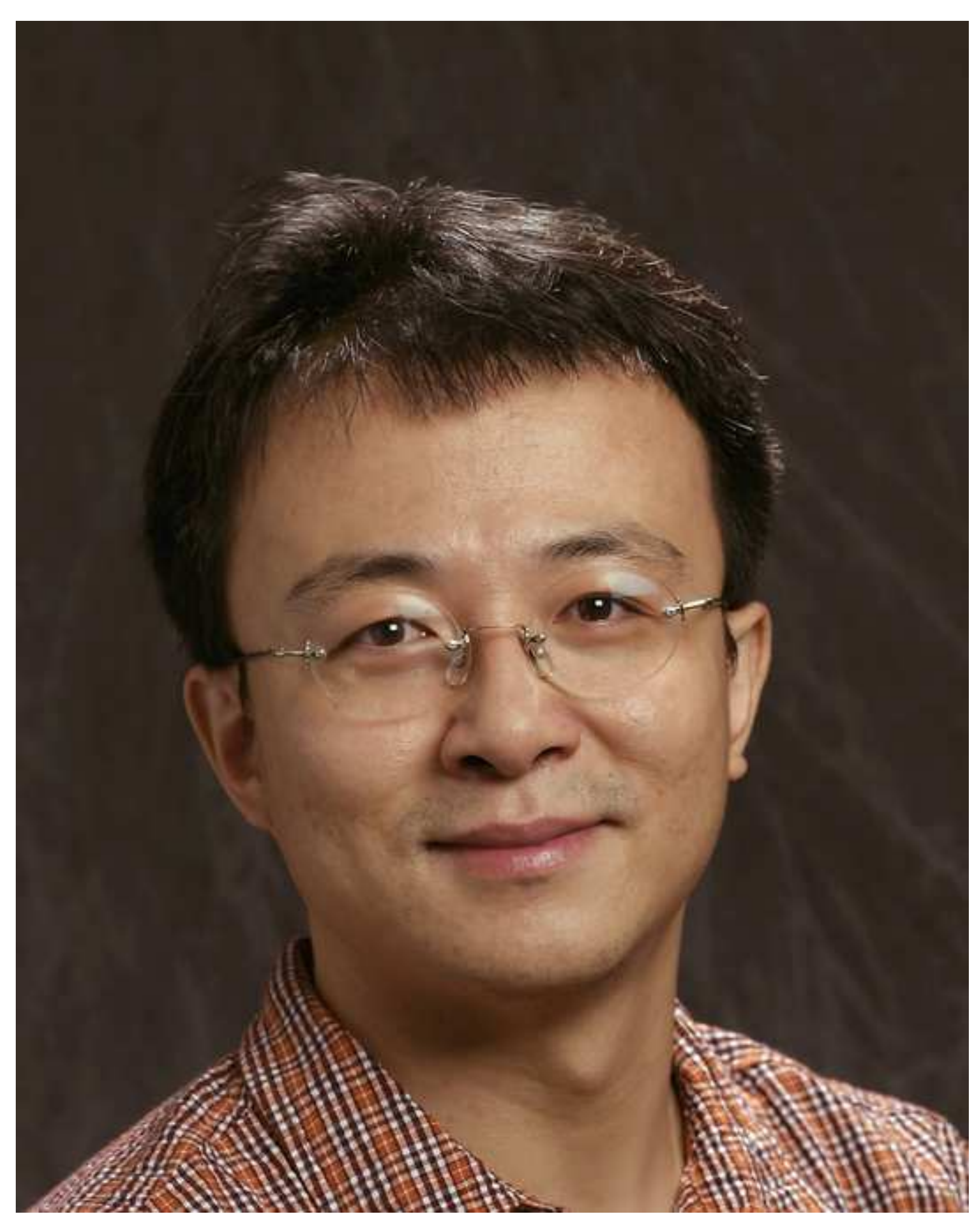

\section{Shuangqing Wei Photo}

$$
\text { . }
$$

.

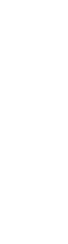

